\title{
Anchor Node Placement for Localization in Wireless Sensor Networks
}

\author{
by \\ Benjamin Tatham, BEng \\ A thesis submitted to \\ the Faculty of Graduate and Postdoctoral Affairs \\ in partial fulfilment of the requirements for the degree of \\ Master of Applied Science in Electrical and Computer Engineering \\ Ottawa-Carleton Institute for \\ Electrical and Computer Engineering \\ Department of Systems and Computer Engineering \\ Carleton University \\ Ottawa, Ontario, Canada \\ January 2011
}

(C) Copyright 2011 - Benjamin Tatham 
Library and Archives

Canada

Published Heritage

Branch

395 Wellington Street

Ottawa ON K1A ON4

Canada
Bibliothèque et

Archives Canada

Direction du

Patrimoine de l'édition

395 , rue Wellington

Ottawa ON K1A ON4

Canada
Your file Votre référence

ISBN: 978-0-494-79554-5

Our file Notre référence

ISBN: 978-0-494-79554-5
NOTICE:

The author has granted a nonexclusive license allowing Library and Archives Canada to reproduce, publish, archive, preserve, conserve, communicate to the public by telecommunication or on the Internet, loan, distribute and sell theses worldwide, for commercial or noncommercial purposes, in microform, paper, electronic and/or any other formats.

The author retains copyright ownership and moral rights in this thesis. Neither the thesis nor substantial extracts from it may be printed or otherwise reproduced without the author's permission.
AVIS:

L'auteur a accordé une licence non exclusive permettant à la Bibliothèque et Archives Canada de reproduire, publier, archiver, sauvegarder, conserver, transmettre au public par télécommunication ou par l'internet, prêter, distribuer et vendre des thèses partout dans le monde, à des fins commerciales ou autres, sur support microforme, papier, électronique et/ou autres formats.

L'auteur conserve la propriété du droit d'auteur et des droits moraux qui protège cette thèse. Ni la thèse ni des extraits substantiels de celle-ci ne doivent être imprimés ou autrement reproduits sans son autorisation.
In compliance with the Canadian Privacy Act some supporting forms may have been removed from this thesis.

While these forms may be included in the document page count, their removal does not represent any loss of content from the thesis.
Conformément à la loi canadienne sur la protection de la vie privée, quelques formulaires secondaires ont été enlevés de cette thèse.

Bien que ces formulaires aient inclus dans la pagination, il n'y aura aucun contenu manquant. 


\section{Abstract}

Applications of wireless sensor network (WSN) often expect knowledge of the precise location of the nodes. One class of localization protocols patches together relativecoordinate, local maps into a global-coordinate map. These protocols require some nodes that know their absolute coordinates, called anchor nodes. While many factors influence the node position errors, in this class of protocols, using Procrustes Analysis, the placement of the anchor nodes can significantly impact the error. Through simulation, using the Curvilinear Component Analysis (CCA-MAP) protocol, we show the impact of anchor node placement and propose a set of guidelines to ensure the best possible outcome, while using the smallest number of anchor nodes possible. Scientists and researchers using sensor networks are thus enabled to focus on the sensed data with confidence in the node localization results. 
Dedicated to my wife and children who supported me through the long process of this research. 


\section{Acknowledgments}

I would like to thank Professor Thomas Kunz for his guidance and encouragement that made this research possible. Further, I would like to thank $\mathrm{Li} \mathrm{Li}$ whom, along with Professor Kunz, performed the initial CCA research and wrote the basis for the Matlab simulations used in this thesis. Finally, my colleagues Shafagh Alikhani and Ben Gardiner who provided a tireless ear to listen to ideas throughout the process. 


\section{Table of Contents}

$\begin{array}{lll}\text { Abstract } & \text { iii }\end{array}$

Acknowledgments $\quad \mathbf{v}$

Table of Contents $\quad$ vi

List of Figures $\quad$ vii

1 Introduction 1

1.1 Motivation . . . . . . . . . . . . . . . . . 2

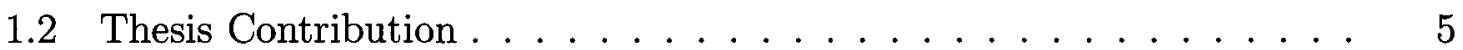

1.3 Methodology . . . . . . . . . . . . . . . . . 6

1.4 Thesis Organization . . . . . . . . . . . . . . . . 7

2 Overview of Wireless Sensor Networks and Localization $\quad 8$

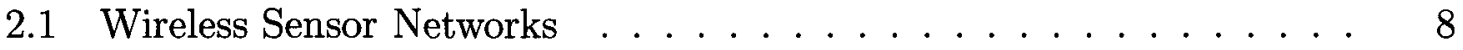

2.2 Localization Protocols $\ldots \ldots \ldots \ldots$

2.2 .1 Ad Hoc Positioning System . . . . . . . . . . . 10

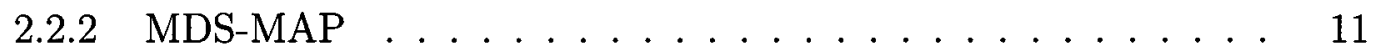

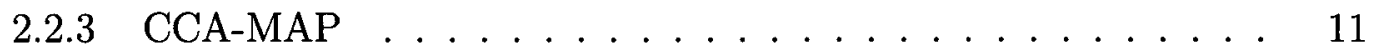

3 Related Work on Anchor Node Placement 13 
$3.1 \quad$ Empirical Evidence . . . . . . . . . . . . . . . . . . . . . . 13

3.2 Explicit Studies of Anchor Node Placement . . . . . . . . . . . 14

3.3 Summary of Related Work . . . . . . . . . . . . . 15

4 Optimal Anchor Node Placements $\quad 16$

4.1 Measuring Location Error _ . . . . . . . . . . . . . . 16

4.2 Coordinate Transformation . . . . . . . . . . . . . 17

4.3 Methodology . . . . . . . . . . . . . . . . 18

4.4 Anchor Node Placement Metrics . . . . . . . . . . . . . . 20

4.4 .1 Anchor Node Error . . . . . . . . . . . . . . 20

4.4 .2 Network Area Coverage . . . . . . . . . . . . 25

4.4 .3 Anchor Node Triangle . . . . . . . . . . . . . . . . 29

4.5 Best Anchor Node Placement . . . . . . . . . . . . . 31

5 Avoiding the Worst Anchor Node Placements 35

5.1 Effects of Procrustes Analysis . . . . . . . . . . . . . 35

5.1.1 Transformation Reflection and Rotation . . . . . . . 37

5.1 .2 Transformation Scaling and Translation . . . . . . . . 39

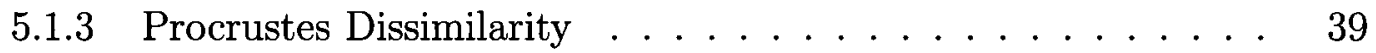

5.2 Outlier Indicators . . . . . . . . . . . . . . . . . . . . . . 43

6 Effects of Network Topology $\quad 47$

6.1 Node Distance from the Anchors . . . . . . . . . . . . . 47

6.2 Applicability to Varying Network Topologies . . . . . . . . . . . . 48

6.2 .1 C-Shape Network Topology . . . . . . . . . . . . . 50

6.2 .2 Pipeline Network Topology . . . . . . . . . . . . . 51

7 Other Factors $\quad 58$ 
8 Conclusion $\quad 62$

8.1 Summary and Contribution $\ldots \ldots \ldots \ldots 2$

8.2 Future Work . . . . . . . . . . . . . . . . . . . . 63

Appendix A Matlab@Simulation Code $\quad 64$

$\begin{array}{ll}\text { List of References } & 64\end{array}$ 


\section{List of Figures}

1.1 Reasonable localization results . . . . . . . . . . . . . 3

1.2 Poor localization results $\ldots \ldots \ldots \ldots \ldots$

4.1 A sample anchor triangle Procrustes analysis . . . . . . . . . . . . 19

4.2 The sample network used in this chapter . . . . . . . . . . . . 21

4.3 Number of anchor neighbors vs. location error . . . . . . . . . . 23

4.4 Mean of anchor node error vs. location error . . . . . . . . . . . 24

4.5 Sum of distances $=\sum_{\imath=0}^{n}\left\|x_{\imath}\right\| \ldots \ldots \ldots \ldots 26$

4.6 Sum of distance between anchors vs. location error . . . . . . . 27

4.7 Minimum distance between anchors vs. location error . . . . . . 28

4.8 Area and height of a triangle . . . . . . . . . . . . . . . 30

4.9 Area of anchor triangle vs. location error . . . . . . . . . . . 32

4.10 Minimum height of anchor triangle vs. location error . . . . . . . 33

4.11 Sum of distance between anchors vs. location error $\ldots \ldots . . . .34$

5.1 Network map of localization performance at each node . . . . . 36

5.2 Rotation and reflection versus location error . . . . . . . . 38

5.3 Transformation scalar component versus location error $\ldots \ldots 40$

5.4 Transformation translation component versus location error $\ldots . . .41$

5.5 Procrustes dissimilarity measure . . . . . . . . . . . . . . 42

5.6 Anchor triangle areas . . . . . . . . . . . . . . . . 44

5.7 Minimum anchor triangle heights $\ldots \ldots \ldots \ldots \ldots$ 
6.1 Localization error as a contour plot . . . . . . . . . . . . . 49

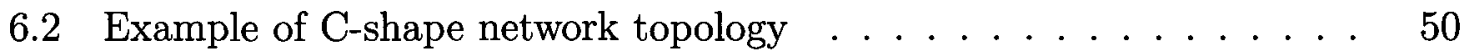

6.3 Anchor metrics for a C-Shape topology . . . . . . . . . . . . 52

6.4 Example of pipeline network topology . . . . . . . . . . . 53

6.5 Sum of distance between anchors vs. mean location error . . . . . . 54

6.6 Pipeline network with anchors spread apart and clumped together . . 55

6.7 Minimum height of anchor triangle vs. mean location error . . . . . 56

7.1 Good localization performance with same anchor set in two different networks 59

7.2 Poor localization performance with same anchor set in two different networks 60 


\section{Chapter 1}

\section{Introduction}

Scientists, engineers, and researchers use wireless sensor networks (WSN) for a wide array of applications. Many of these applications rely on knowledge of the precise position of each node. While some may only require relative coordinates within the network, most biological, geophysical, and other scientific applications require coordinates on a global coordinate system. Perhaps the obvious solution is for each node in the network to be equipped with GPS or other location positioning services. However, constraints on cost, power consumption, as well as visibility of satellites dictate the need for an alternative solution.

Many protocols have been proposed [?,?,?] to calculate relative positions amongst the nodes of a network. They vary in the required network functionality in terms of radio ranging or range-free. Radio ranging involves specialized hardware to measure the distance between nodes based on physical data like signal strength or transmission delays. Procrustes analysis [?] is a common method to convert from relative to global coordinates, requiring some of the nodes to have a local source of global coordinates. This can be achieved by operators recording the global coordinates during network deployment, by embedding a GPS receiver in a subset of the nodes, or some other source. We call these enhanced nodes anchors. Here, we explore the effect of anchor 
node placement within the network on the overall localization errors, on a networkwide basis. This provides network planners with a set of general rules to minimize the number of anchor nodes required while avoiding poor node localization, allowing scientists to assume a maximum position error during their own research. Further, based on application requirements of location accuracy, planners can minimize the cost of the network associated with anchor nodes by using the minimum number and best position.

\subsection{Motivation}

During previous work designing localization protocols, researchers often choose anchors at random within the network [?, p.11] [?, p.2]. Sometimes, they simulate the network multiple times with different anchors in order to statistically exclude anchor node placement from their results.

Our initial investigations and simulations demonstrate that indeed the placement of anchor nodes in the network does have an often dramatic effect on the location error. The four plots shown in Figures 1.1 and 1.2 graphically establish that anchor node position does make a difference. Each plot shows the same network with a different choice of three anchors. A line is drawn between the actual and calculated position of each node to visualize the localization error. The circles show the radio range of each anchor, and a triangle is drawn between the three anchors for clarity.

The four plots shown are taken from a set of 100 randomly chosen anchor sets. While the first two choices, shown in Figure 1.1, have reasonable errors, the third choice, in Figure 1.2a, has an error more than twice the mean error of the first. Further, the fourth choice, in Figure 1.2b, has an extremely poor performance, more than ten times that of the first choice. The four anchor set triangles shown do not 


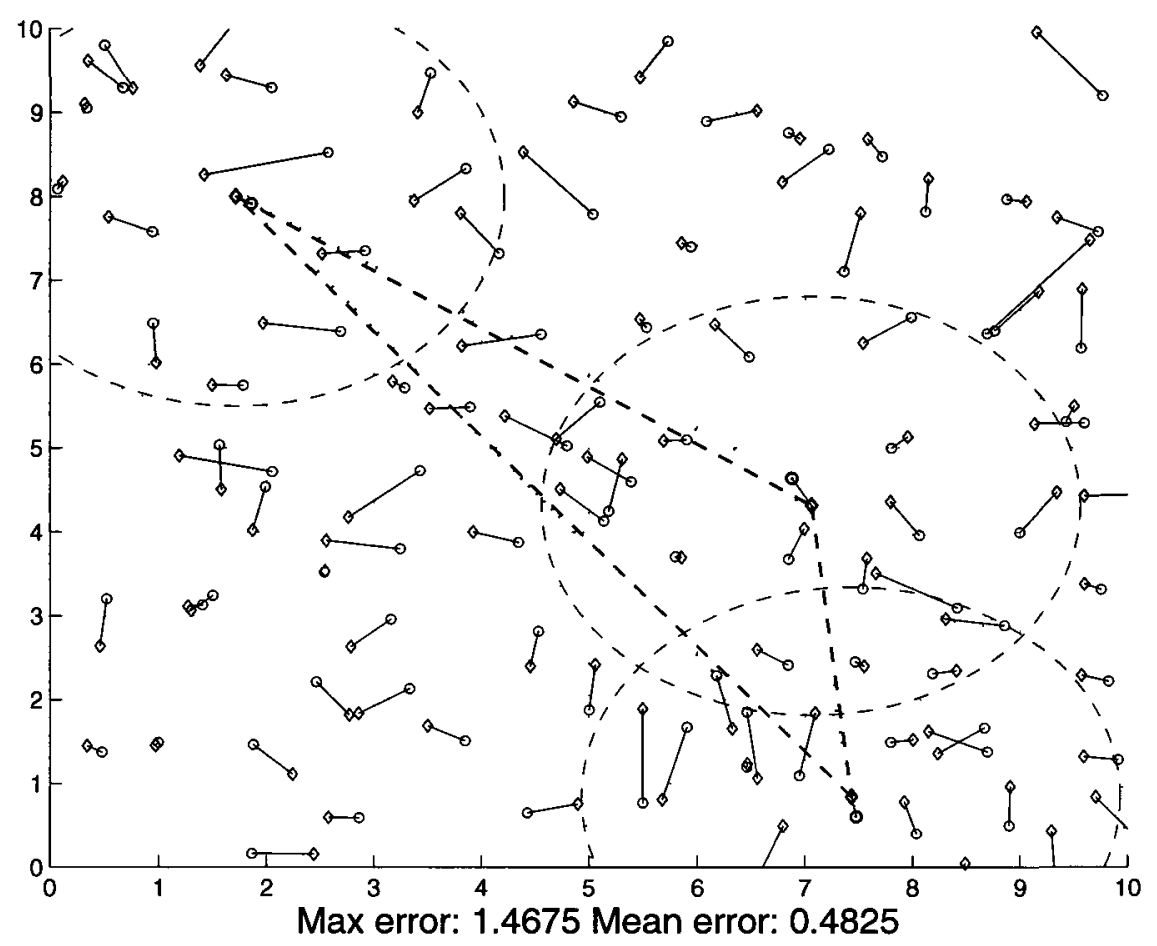

(a) Anchor Set 1

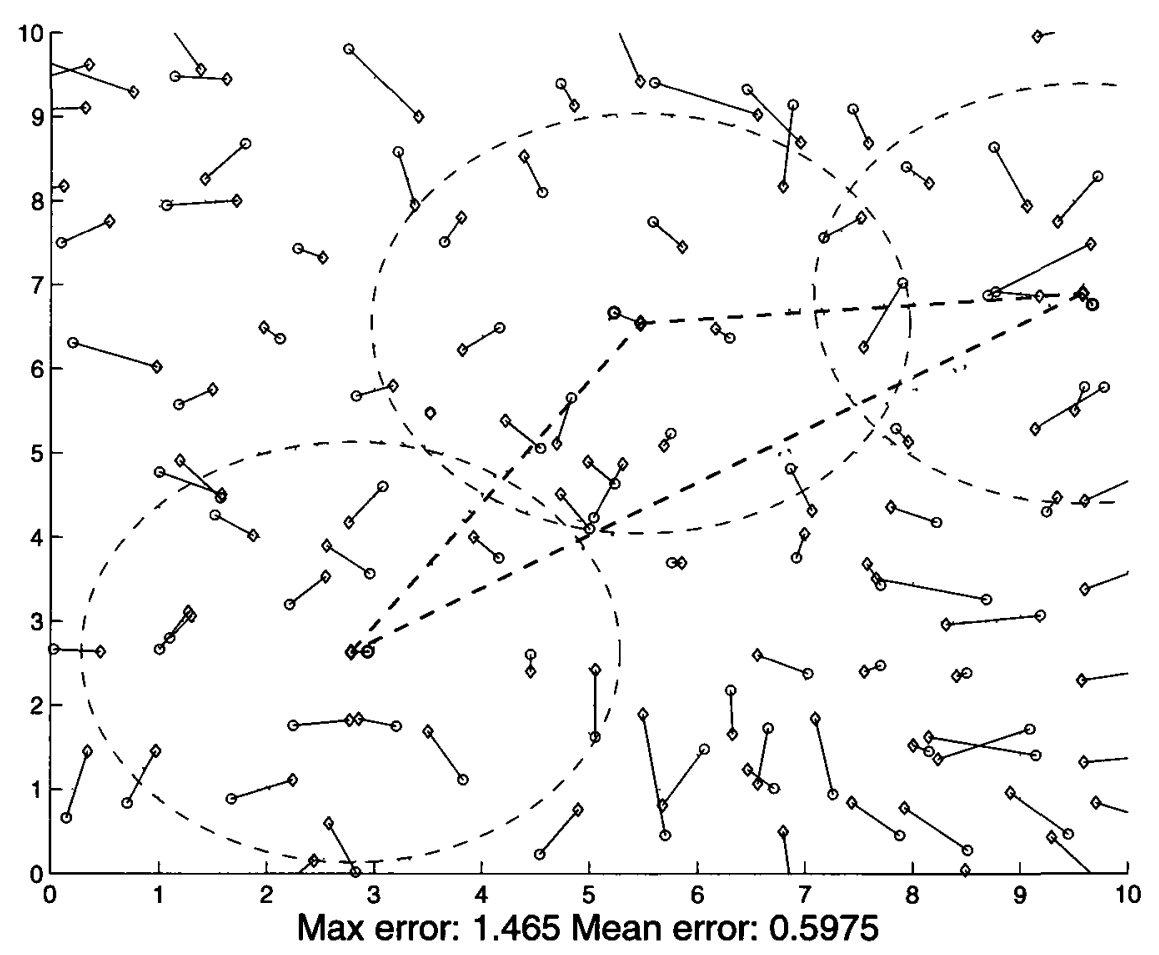

(b) Anchor Set 2

Figure 1.1: Reasonable localization results 


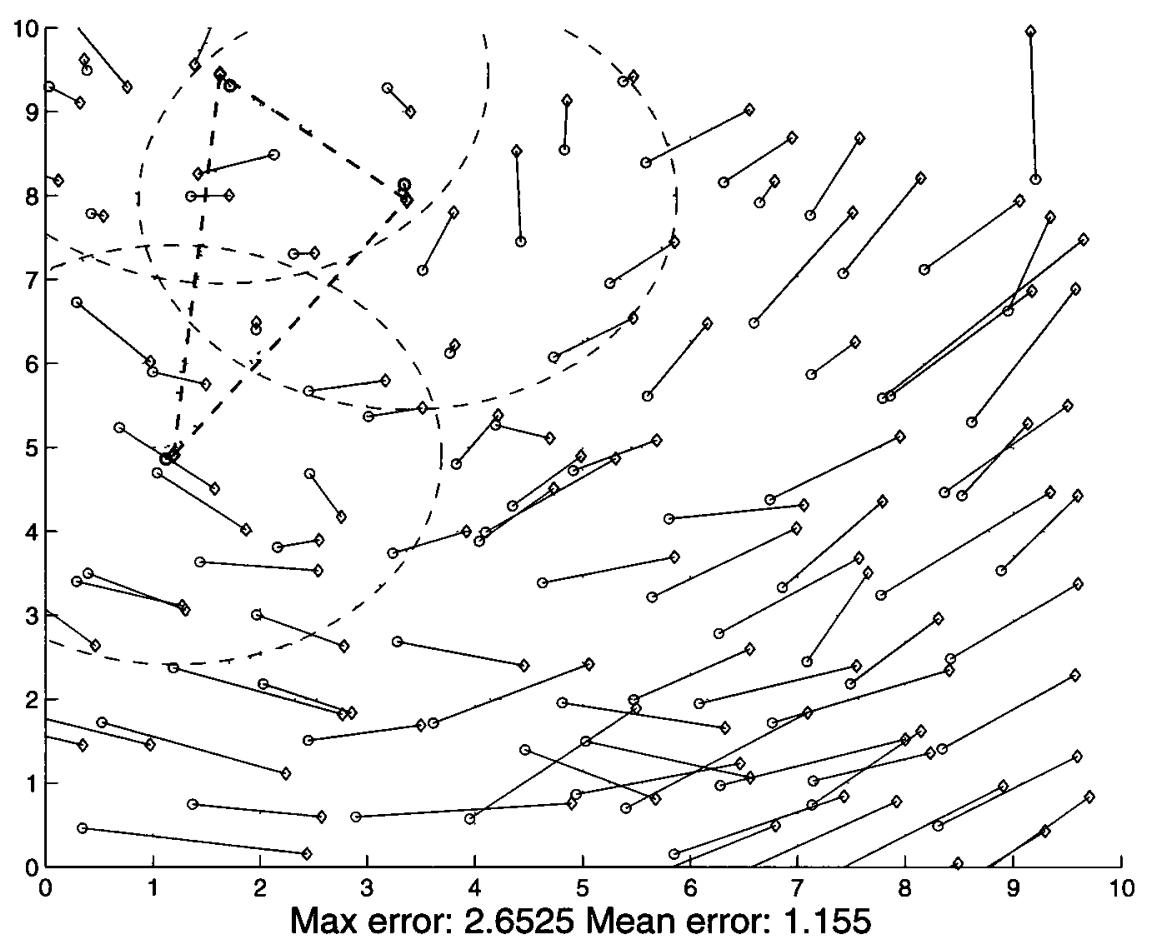

(a) Anchor Set 3

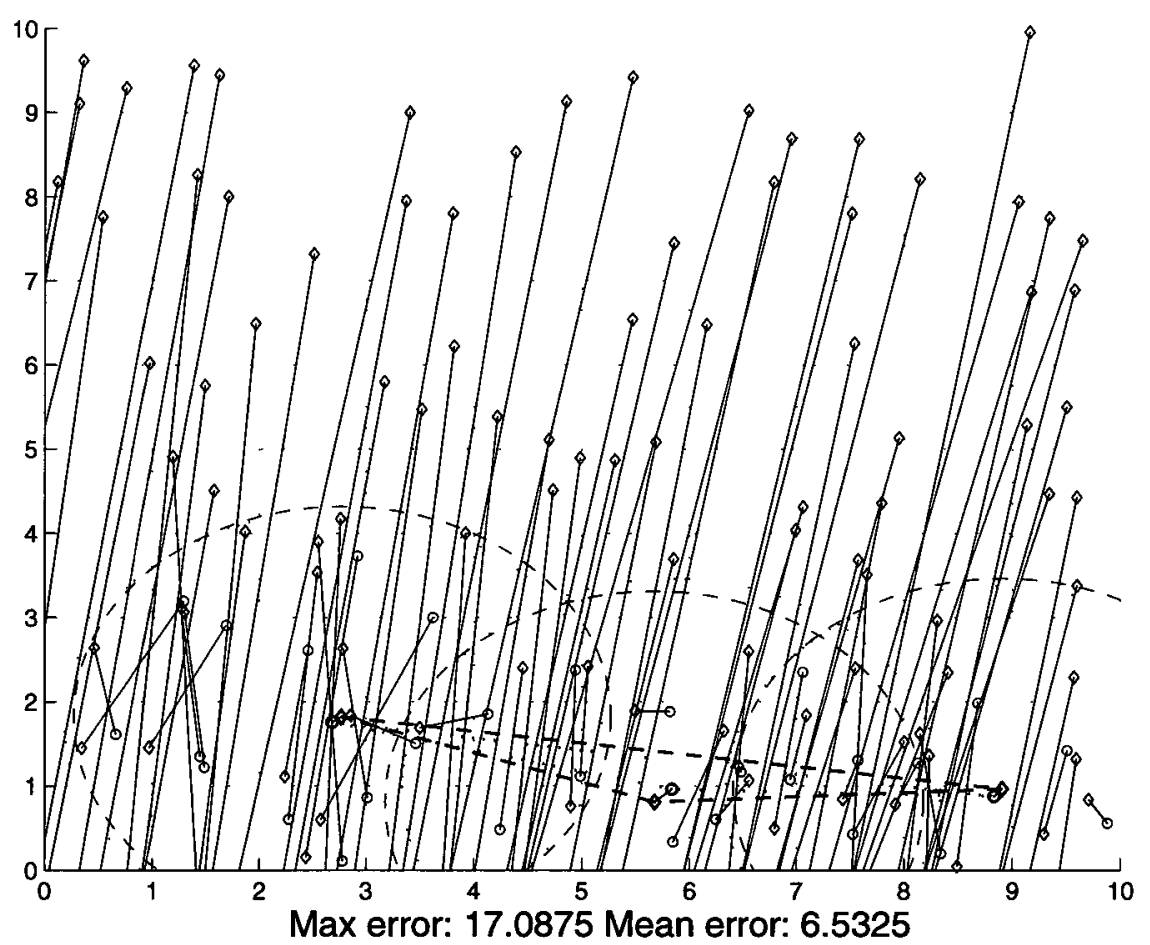

(b) Anchor Set 4

Figure 1.2: Poor localization results 
immediately show an obvious progression that could explain this dramatic change in error. We find it interesting that there is an incremental increase in error between these four anchor sets resulting in a full order of magnitude difference between good and bad cases, and thus requires the investigation presented in this thesis.

Since there is a chance that the localization errors can be large due to anchor node placement, scientists and engineers need to know what degree of accuracy the localization algorithm provides. In practical terms, this information can be useful in many cases. First of all, if a network designer has an existing sensor network or is planning to deploy one, they need to know where to place the anchor nodes to ensure localization accuracy. While the freedom of where to place the anchor nodes may be constrained due to physical factors, the network designer still must be able to choose anchor placements wisely. Secondly, even if the network is already deployed, the guidelines can provide confidence that the localization results are good enough and the research resulting from the sensed data itself is valid with respect to location of the sensed data.

\subsection{Thesis Contribution}

Although some papers have touched upon anchor node placement, we have yet to come across a comprehensive study of the anchor node placement using Procrustes analysis. This thesis provides a comprehensive study of possible anchor node placements and their effects on overall network localization accuracy.

Specifically, this thesis establishes two guidelines based on metrics that a user of a wireless sensor network can calculate at any time during network planning or deployment, based on information readily available to them. These guidelines provide a way to exclude extremely poor localization results and ensure that the localization 
results fall within a range of errors that are statistically insignificant. We do not address a variety of other possible factors that may may also influence localization error, although these are discussed briefly in Chapter 7 .

At a high level, the guidelines are a result of the fact that the probability of extremely high location error results from anchor nodes being roughly in a geographically straight line. As the anchor nodes are spread out from a straight line, the probability of high errors decreases, leaving network designers a relatively simple chore when choosing anchor nodes locations.

While this thesis is a comprehensive study of anchor node placements when using Procrustes analysis, we focus on a specific set of network design assumptions. First, we assume the network planners want to minimize the number of anchors since they consume more power and cost more. Therefore, we use the minimum number of anchors of three to provide two-dimensional coordinates. Further, we assume that range-free algorithms are preferred to avoid dealing with multi-path and other radio effects as well as to limit the required hardware and power requirements on each node in the network. For that reason, our simulations use range-free techniques. Nonetheless, the guidelines presented here are agnostic to whether the underlying localization algorithm uses range-based or range-free techniques. Finally, we assume that the network density is sufficient to provide a fully connected network, meaning that there is a single connected graph of all the nodes in the network.

\subsection{Methodology}

Many localization protocols and algorithms provide a set of relative coordinates that are then transformed into global coordinates. For the purpose of this research, we chose CCA-MAP [?, ?] as the algorithm to provide simulation results. 
A Matlab@ simulation of this algorithm already existed [?], and was modified to provide the necessary output statistics presented here. CCA-MAP is described in more detail in Section 2.2.3.

\subsection{Thesis Organization}

A brief background of Wireless Sensor Networks and localization protocols in general are presented in Chapter 2. Chapter 3 presents the limited related work on anchor node placement. Chapter 4 contains various proposed anchor node placement methods and summaries of how they perform. The cause of the extreme edge cases comes to light in Chapter 5. Chapter 6 discusses various other factors. Chapter 7 gives some ideas for future work, including other factors that effect localization error and Chapter 8 summarizes the results of this study. 


\section{Chapter 2}

\section{Overview of Wireless Sensor Networks and Localization}

\subsection{Wireless Sensor Networks}

A wireless sensor network (WSN) consists of a set of nodes tasked with sensing environmental phenomenon at or near each node. Nodes communicate via radios to send their data back to a central acquisition system. Nodes are typically small, cheap devices and are designed with power efficiency in mind to prolong the lifetime of the network's ability to collect data. Nodes are often distributed in the field of interest randomly, sometimes even by dropping them from the air, as on a military battlefield. Other times, they are placed in specific, but unknown a priori, locations, as in placing them in bird nests [?]. Or, they may be rolled into a transportation tunnel to give firefighters and emergency crews current information about heat and oxygen levels [?].

A number of issues arise when designing a WSN. Each node must be able to communicate with other nodes and send data to a central collection site. Each node must know what time it is, for purposes of data sampling, and often for routing 
protocols as well. Further, each node must know where it is so spatial data can be properly correlated. Location can also be useful for geographic routing protocols. This thesis focuses on determining the location of each node as accurately as possible.

\subsection{Localization Protocols}

There are two general classes of localization protocols: ranging and range-free. Ranging protocols rely on information from the radio. With this information, a fairly accurate network topology can be built. Ranging techniques can use a variety of metrics to build the network topology. These include Time-of-Arrival (TOA), like GPS [?], Time-Differential-of-Arrival (TDOA) [?], Angle-of-Arrival (AOA) [?], or Received-Signal-Strength-Indicator (RSSI) [?].

However, the special hardware and power requirement to perform these ranging techniques is counter to the goal of low-cost, low-power nodes, and thus we exclude ranging protocols from our study. Regardless, if a ranging protocol does build a relative map, and then does a post-processing step by mapping this relative map to a global map based on a subset of anchor nodes, the results of this thesis apply to ranging as well as range-free protocols.

Range-free protocols do not rely on any specialized hardware for additional information. Rather, they rely solely on network connectivity, specifically knowledge of their direct neighbors. Often, a node will collect information about their direct neighbors' neighbors as well, known as two-hop information. Knowledge of each further node requires more information to be shared and therefore transmitted between nodes, thus requiring more power for radio transmission. It is for this reason that only one-hop or possibly two-hop knowledge is preferred. 


\subsubsection{Ad Hoc Positioning System}

Niculesu, et al. propose a distributed localization algorithm known as Ad Hoc Positioning System (APS) [?]. It is similar to GPS in that is uses triangulation to determine node positions. In APS, each node maintains a table of distances to each anchor. The distance can be represented as a hop count, estimated distance using RSSI, or Euclidean distance. As a distributed algorithm, each node determines its own position based on the distances to the anchor nodes. Thus, APS does not perform well in anisotropic network, that is networks with holes or "C" shapes in the topology, because the communication distance can be far greater than the geometric distance between two nodes.

In its simplest form, APS uses a propagation technique called DV-HOP to determine distances between nodes. DV-HOP is based on classical distance vector exchange from general network protocols like TCP/IP. Each node maintains a table of hop counts between all known nodes. Each node exchanges this table only with its direct neighbors. When an anchor has discovered a hop count to another anchor, the anchor estimates the average distance for each hop since it knows the absolute location of itself and the other anchor. This correction factor is sent to the entire network. DV-HOP thus minimizes the amount of data that must be transmitted in the network.

Further, APS can employ a propagation technique called DV-distance. DVdistance is similar to DV-hop except that it uses RSSI to determine each hop distance and sends this distance instead of hop count. This difference allows DV-distance to effectively detect holes and curves in the network as each anchor can see that the transmission path between them is larger than the Euclidean distance. 


\subsubsection{MDS-MAP}

Shang, et al. attempt to correct the errors introduced by APS and other distributed algorithms through a centralized localization algorithm called MDS-MAP(C) [?], where the $C$ is for centralized. MDS-MAP $(\mathrm{C})$ is divided into three phases. In phase one, shortest path distances or hop counts are exchanged via a distance vector exchange, similar to APS. This provides a rough estimate of the distance between each pair of sensors. In phase two, multi-dimensional scaling (MDS) is applied, resulting in a relative map. MDS is a general data analysis tool originating from psychophysics [?, p.2] to transform data from many to few dimensions. In simple terms, MDS takes a set of distances between points and creates a structure that fits those distances. Often, it is used for general data visualization. In this case, the relative map conforms closely to the pair-wise distances provided. In phase three of MDS$\operatorname{MAP}(\mathrm{C})$, the relative map is transformed into a global coordinate system using at least three anchors using the Procrustes algorithm. Procrustes is described in more detail in Section 4.2 .

The authors provided a modified, distributed version, $\operatorname{MDS}-\mathrm{MAP}(\mathrm{P})$ [?]. This variation simply divides the network into smaller, more manageable sections so that the algorithm can be performed locally, with the limited node resources available. Each local map is then patched together, and hence the $P$ for patched. The patching part of the algorithm is not distributed. Local map merging begins at a randomly selected node's local map, and chooses the local map with the most overlapping nodes. The process continues until all the local maps are merged together.

\subsubsection{CCA-MAP}

$\mathrm{Li}$, et al, propose a similar style algorithm to MDS-MAP called CCA-MAP [?, ?]. It is similar in that it generates relative, local maps of sections of the network and 
then patches them together into a global coordinate system. CCA-MAP improves on MDS-MAP in that the algorithm is more efficient. MDS is a non-linear reduction algorithm and has a computational cost of $O\left(n^{3}\right)$, where $n$ is the number of nodes in each local map. The size of each local map is dependent on the radio range which affects the number of neighbors for each sensor node. Further, the algorithm could be run in a centralized way, which means that $n$ becomes the total number of nodes in the network. CCA [?] on the other hand, is a self-organized neural network performing quantization and non-linear projection. CCA-MAP has a total computational cost of $O\left(n^{2}\right)$. CCA runs in a series of iterations, where each iteration has a computational cost of $O(n)$.

CCA-MAP has four phases. In the first phase, each node builds a local map of nodes within $R$ hops. For that local map, the shortest distance matrix is accumulated, as in APS and MDS-MAP. The second phase involves performing the CCA algorithm itself on each local map, generating relative coordinates for each node in the local map. In phase three, the local maps are merged together, as in MDS-MAP(P), and finally, in phase four, the relative coordinates are transformed into absolute coordinates based on the known coordinates of the anchor nodes, as described in Section 4.2. Phase four can only be performed with a minimum of three anchors for $2 D$ space or four anchors for $3 D$ space.

CCA-MAP is flexible as to where computations can be performed. Local map calculations can be performed at the nodes themselves, if computing resources allow, or outsourced to more powerful gateway nodes or a central server. Local map merging can be performed in parallel at selected nodes in the network, or again at a central server. Further, if in any sub-map sufficient anchors are found, then absolute coordinates can be calculated. 


\section{Chapter 3}

\section{Related Work on Anchor Node Placement}

While much attention has been paid to localization accuracy and computational effort, the importance of intelligent anchor node placement is often recognized, but dismissed as future study.

\subsection{Empirical Evidence}

Often, authors will come across anchor placement by accident and discuss it based on their own empirical evidence. Shang, et al. [?, p.964] and Li, et al. [?, p.11] both choose anchors at random within the network. Although, Shang, et al., do mention that a co-linear set of anchors chosen in one example "represents a rather unlucky selection", without supporting evidence of why this is unlucky.

Earlier work by Doherty, et al. [?] requires anchor nodes to be placed at the edges, and ideally at the corners of the network. In this case, however, the algorithm is a simple constraint problem. One constraint requires that all the unknown nodes be placed within the convex hull of the anchors, and therefore, better results are obtained when anchors are at the corners. 


\subsection{Explicit Studies of Anchor Node Placement}

While few, there have been a some explicit studies of anchor node placement. Hara, et al. [?], propose a method of choosing anchor node locations to achieve a specific accuracy target. The proposal, however, only applies to rectangular network areas and that anchor nodes must be placed at the center of a sub-rectangle of the original rectangle when divided into equal sized rectangles. Further, it assumes simple RSSIbased localization.

Ash, et al. [?], provide analytical proof that placing anchor nodes uniformly around the perimeter of a network provides the best results, in the absence of any other information about the sensor node positions. However, again this assumes a rectangular network, and more importantly a simple localization algorithm like [?] or other multilateration techniques. When using all inter-node distances at once, as in MDS-MAP and CCA-MAP, this analysis breaks down.

Karl and Willig dedicate an, albeit short, sub-chapter to the Impact of anchor placement in their book [?, p.247-248]. Referencing [?] and [?], again they defer to perimeter anchor placement as the optimal choice. Unfortunately, the technique proposed involves adaptive deployment, whereby a mobile node with absolute positioning available, like GPS, wanders through the network and attempts to determine the optimal anchor placements as it travels. For the purposes of a priori planning, this technique is not feasible.

Cheng, et al. [?] present a novel technique to handle the effects of adverse anchor placement, specifically in clumps. The algorithm, HyBloc, is a hybrid of MDS and proximity-distance map (PDM) [?]. HyBloc combines the two algorithms to draw on the best aspects of each. Namely, PDM is shown to have good performance in anisotropic networks, while MDS performs well even with few anchors. Anisotropic 
networks have irregular shapes or holes in the network connectivity. HyBloc, therefore, works by using MDS to add artificial, secondary anchor nodes in specific isotropic areas of the network, and then uses PDM to complete the overall localization for the entire network.

Another study focuses on the effect of indoor conditions and anchor placement as it relates to RSSI and other radio propagation measurements [?]. The experiments were conducted in a small, enclosed space, and anchor nodes were placed either on the ceiling or the floor of the room. The study concludes that anchor nodes on the ground are better for monitoring moving people in the room, the extension of which is that anchor nodes need to be in the same plane as the nodes they are being used to locate.

\subsection{Summary of Related Work}

Overall, the previous studies on anchor placement are limited. Specifically, they focus on particular use cases and assumptions that are different from the ones here. The overarching theme of the studies, though, is to place the anchors at the edges of the network. Despite the different assumptions, this idea makes sense from a purely geometric point of view, and is therefore used as the initial basis of hypotheses in this thesis. 


\section{Chapter 4}

\section{Optimal Anchor Node Placements}

\subsection{Measuring Location Error}

Before searching for the best anchor node placement we must first define what best means, in terms of location error. First of all, location error is the distance between the actual and calculated position of each node, measured as a factor of radio radius (or range). Since this study addresses range-free networks, and thus relies solely on network connectivity, the actual units of distance do not matter for general study. What is important to the protocol is how many other nodes in the network fall within the radio radius of a given node. The average number of nodes within range of each node is known as network density.

Every network has its own application requirements, and thus there are many options for what statistics to examine for accessing the quality of locations and therefore what best means. The simplest criteria is to look at the mean location error across all nodes in the network. This is the basis for the results in this study. However, this assumes that all nodes in the network must be used in the final results. If the network designers know which nodes have poor locations, they may wish to exclude 
these nodes from the final results. Therefore, if the economics of the network deployment allow, it may be beneficial to look at the best, for example, $80 \%$ of nodes in the network. In practice, the designers do not know which nodes to exclude, so this study also attempts to identify a correlation between a particular node's position relative to the anchor nodes and its localization error. However, no such correlation was determined in our studies.

Other metrics to consider would the minimum or maximum location error in the network. In both of these cases, it isolates a single, arbitrary node from the entire network as the key for the results. The node with minimum error would almost always be an anchor node or a sensor node very close to an anchor node, further skewing the results. Therefore, while we could use other metrics to determine the best anchor placement, we use the mean location error of all nodes in the network. This hopefully provides the most inclusive and comprehensive result.

\subsection{Coordinate Transformation}

To understand the various hypotheses for the best anchor placement, a basic understanding of how the transformation from local to global coordinate systems is required. After the local networks have been calculated and patched together into a single cohesive network, the anchor nodes are then used. Using Procrustes [?] analysis, a linear transformation of translation, reflection, orthogonal rotation, and scaling is determined for the anchor nodes from the calculated to known coordinates. The transformation is chosen by minimizing the sum of squared errors for the resulting coordinates. Specifically, given actual anchor coordinates, $Y$, Procrustes gives a transformation as shown in Equation 4.1 that minimizes the difference between the actual 
coordinates and the calculated coordinates, $Z$. In the equation, $b$ is a scalar component, $T$ is the rotation/reflection component, and $c$ is the translation component, each determined by the Procrustes analysis. The rotation/reflection component, $T$, is discussed in more detail in Section 5.1.1.

$$
Z=b * Y * T+c
$$

For example, take a random anchor set, as shown in Figure 4.1. The figure shows the three triangles, which are the local anchor coordinates, the actual global coordinates, and the predicted coordinates after applying the calculated transformation from local anchor coordinates to actual anchor coordinates. This demonstrates that the transformation calculated by Procrustes is not perfect, as the actual and calculated coordinates do not align exactly.

The transformation is then applied to all nodes in the network by simply replacing $Y$ in Equation 4.1 with the local coordinates for the entire network. Therefore, how well the anchor nodes transformation captures the overall local network variability dictates how good the final locations will be.

\subsection{Methodology}

In order to assess anchor node placement, a series of hypotheses are presented. Each hypothesis is centered around a metric that can be calculated from the anchor nodes themselves, or other data the network designer might have before deploying the network or when analyzing the localization results. In other words, none of the hypothesis would require extra information that must gathered about the sensor nodes or their actual locations. 


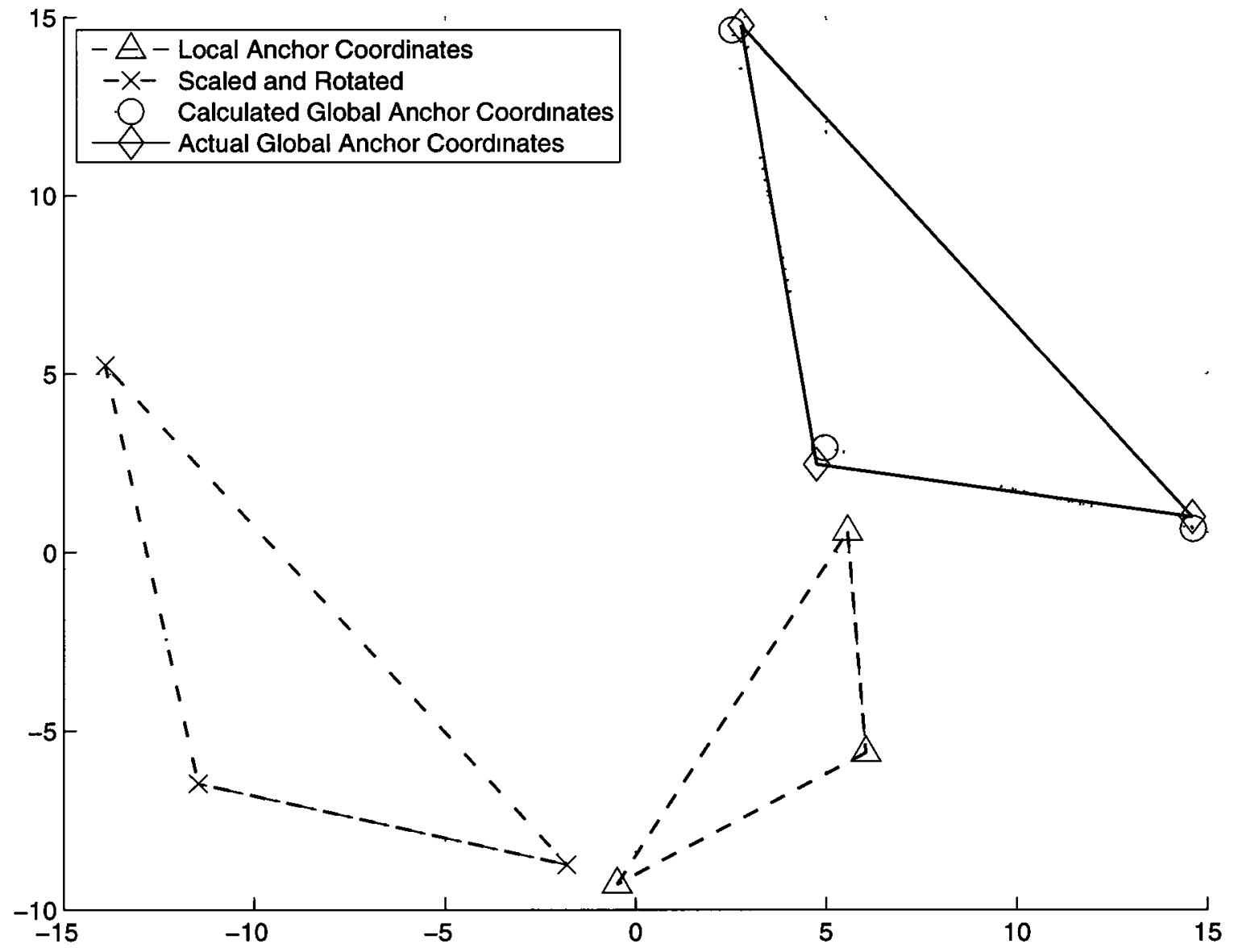

Figure 4.1: A sample anchor triangle Procrustes analysis 
For the purposes of testing each hypothesis, randomly generated networks of varying sizes are used. Unless otherwise specified, all nodes are randomly placed within a square area with an overall density of one node per unit area. For example, a 20x20 square area will have 400 randomly placed nodes. Anchor sets are chosen by identifying all possible sets via $n$ choose $k$, where $n$ is the number of nodes in the network and $k$ is the number of nodes per anchor set. For example, in a 20x20 network of 400 nodes, and three nodes per anchor set, there are a possible 10,586,800 choices. From the total set of possible anchor sets, a random selection is made. For the data in this chapter, 1,000 anchor sets are randomly chosen. Choosing anchor sets from the $n$ choose $k$ population excludes the possibility of choosing the same anchor set more than once. To view all the Matlab(c) code for simulations and plots see Appendix A.

\subsection{Anchor Node Placement Metrics}

Almost as important as knowing which factors effect localization is knowing which factors to ignore. The following is a summary of hypotheses of anchor node placement factors that show no or little significant correlation to location error. The data in the following graphs is taken from a random selection of 1000 anchor sets of three nodes each for the network shown in Figure 4.2. The node placement is random within the 20 by 20 square unit area, and all nodes have a radio range of 2.5 units, providing a completely connected network.

\subsubsection{Anchor Node Error}

The first hypothesis is to choose nodes as anchors that we expect to have high location accuracy. Because we are trying to provide an a priori design technique for network planners, the information chosen to reach this goal must be something the network 
Random $20 \times 20$ Square with 400 nodes Radio Range 25

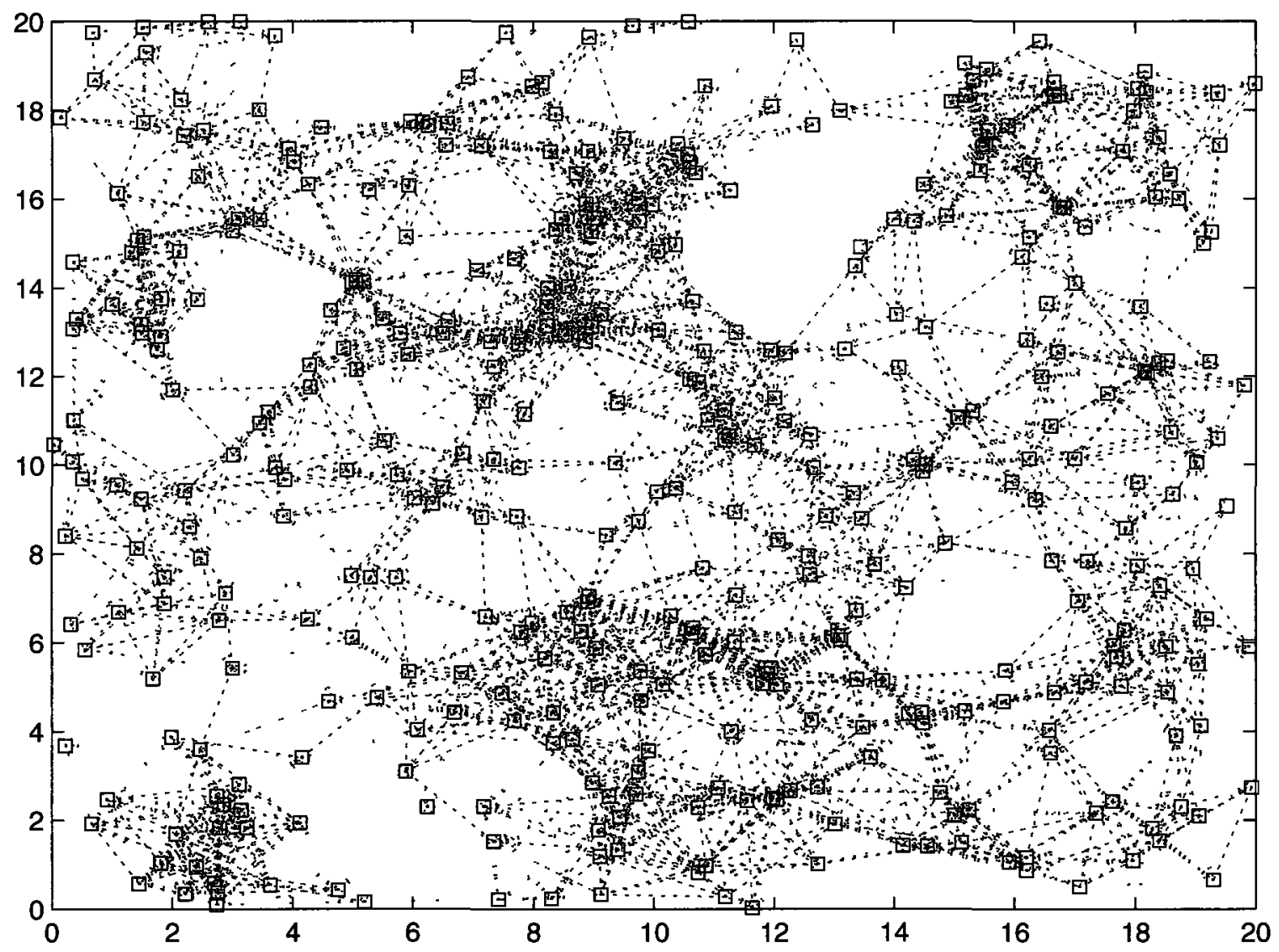

Figure 4.2: The sample network used in this chapter, showing connectivity between nodes 
planner can determine before any localization has been performed. For this purpose we choose the number of one-hop neighbors. On average, increased network density results in higher localization accuracy [?,?]. Since the range-free algorithms depend heavily on network connectivity, the theory is that nodes with more neighbors will have lower location errors. The lower error in the anchors themselves should then translate into a more accurate transformation of the entire network.

Figure 4.3a plots the sum of unique one-hop neighbors for all anchor nodes versus mean localization error. The irregularity of the curve disproves the hypothesis. Further, the correlation coefficient, with $95 \%$ confidence intervals, is extremely low, less than 0.1. Based on these statistics, choosing anchors in denser parts of the network does not translate into better network-wide localization accuracy.

We also explore if having anchors with lower localization error translates into lower localization error across the network. Figure $4.4 \mathrm{a}$ plots the mean error of the anchor nodes themselves versus the mean error for all nodes in the network. This essentially measures how well the transformation generated by Procrustes analysis matches the target. The erratic plot and low correlation coefficient suggests there is no foundation for this hypothesis.

In both plots, it is clear that a small subset of the anchor placements lead to far greater error than the norm. Later, we will explore the cause of these outliers, but for the time being, we exclude them from the analysis as shown in Figure $4.3 \mathrm{~b}$ and Figure 4.4b. Determining which data points are outliers is quite obvious since the gap between the normal case and the small set of outliers is distinct. Therefore, rather then using a statistical calculation for the exclusion, all anchor sets with mean location error greater than two are excluded.

Even after the outliers are excluded, no pattern emerges and the correlation coefficients remain extremely low. Further, the line of best fit has a low r-squared value 


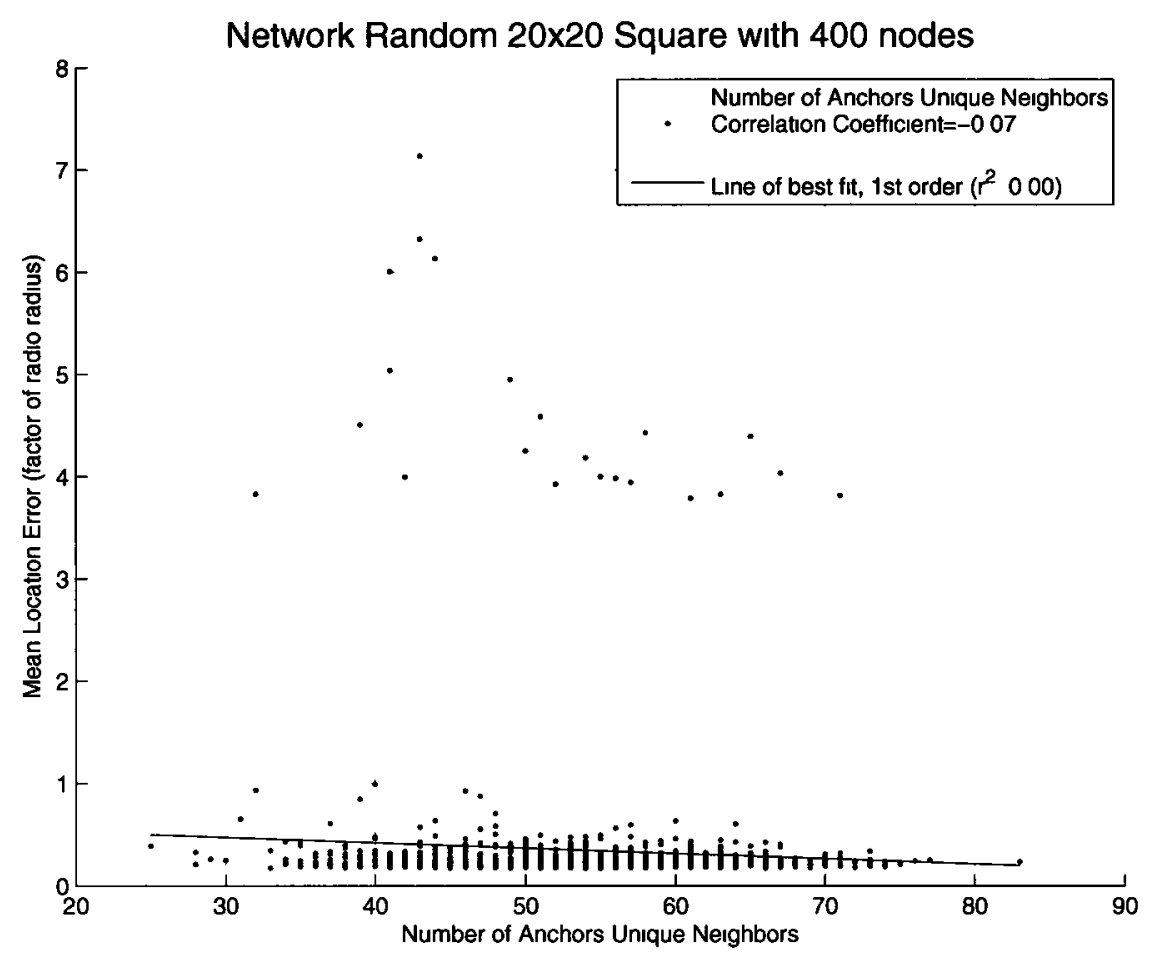

(a) All anchor sets

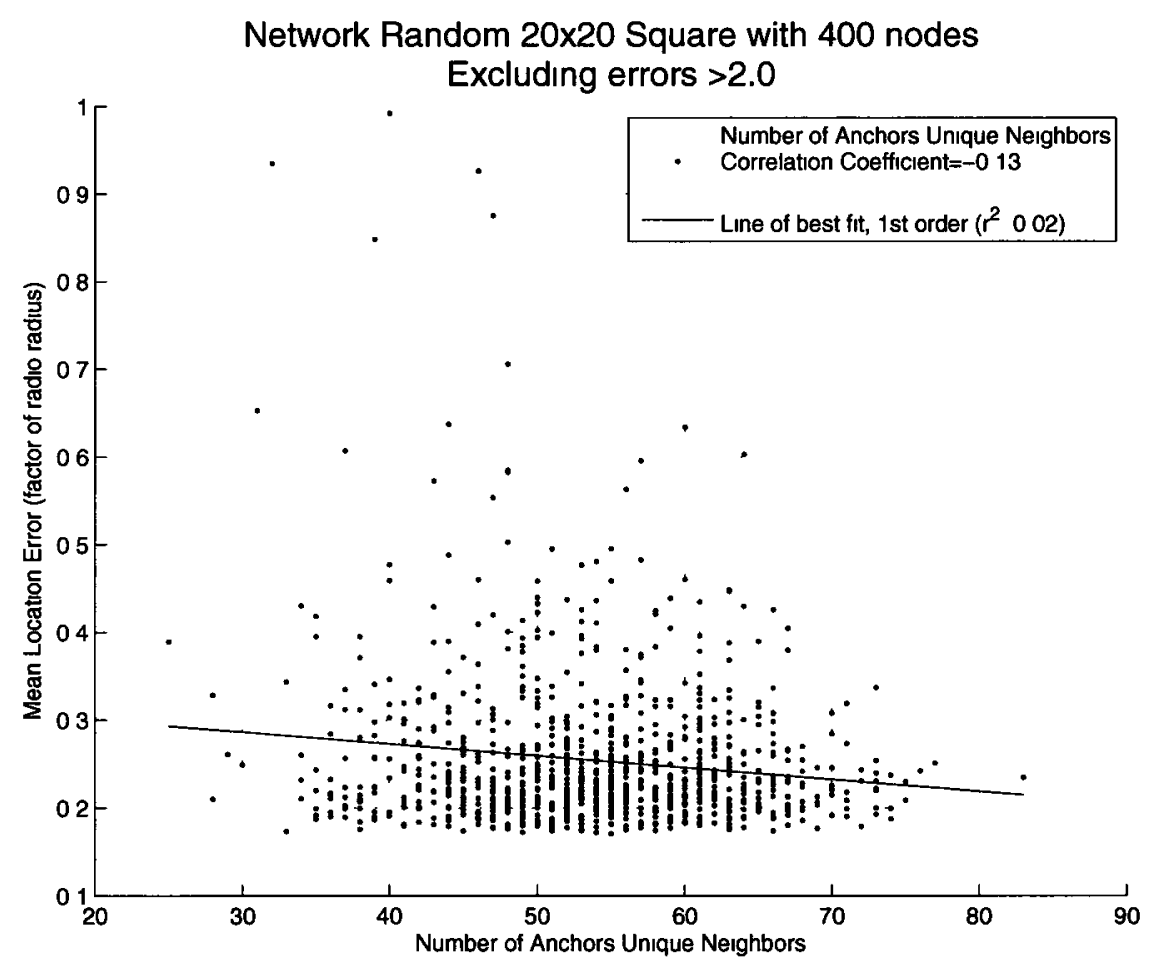

(b) Excluding outliers

Figure 4.3: Number of anchor neighbors vs. location error 


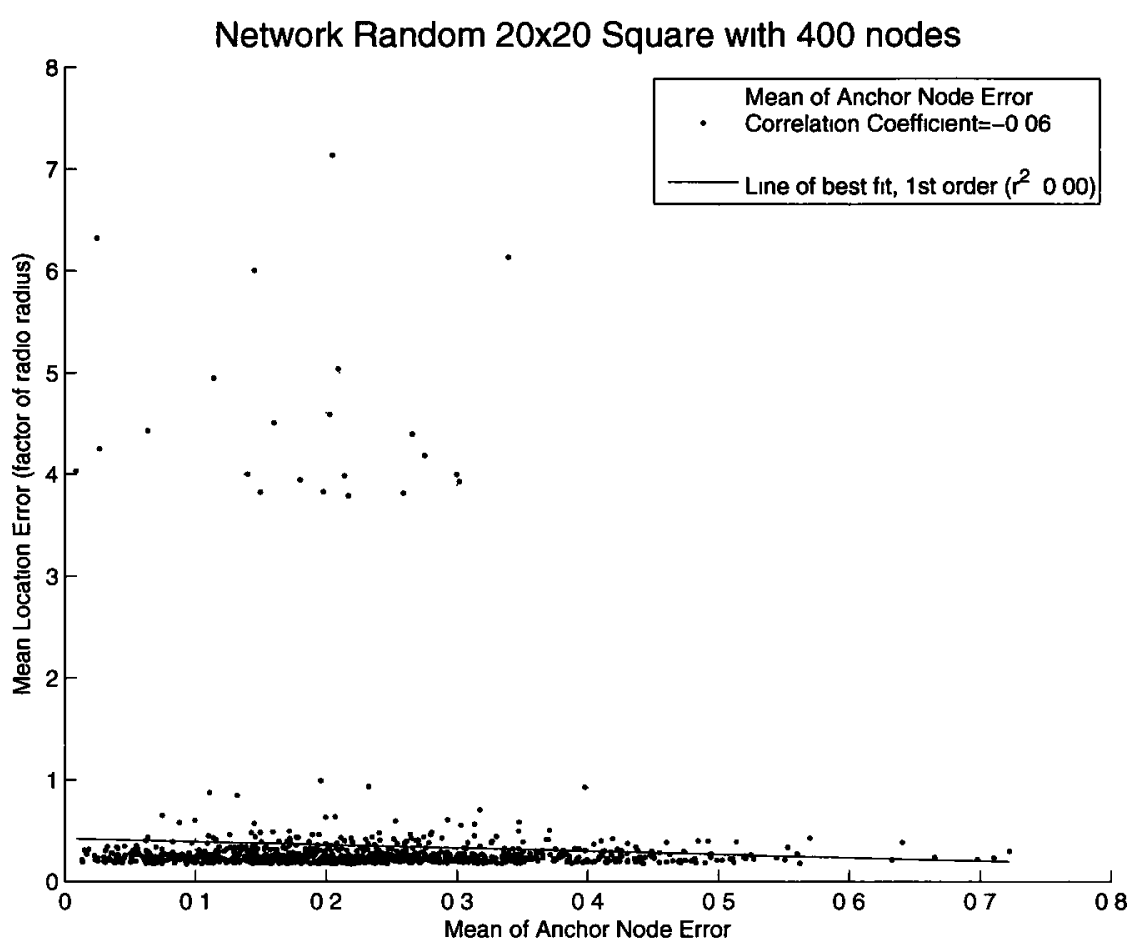

(a) All anchor sets

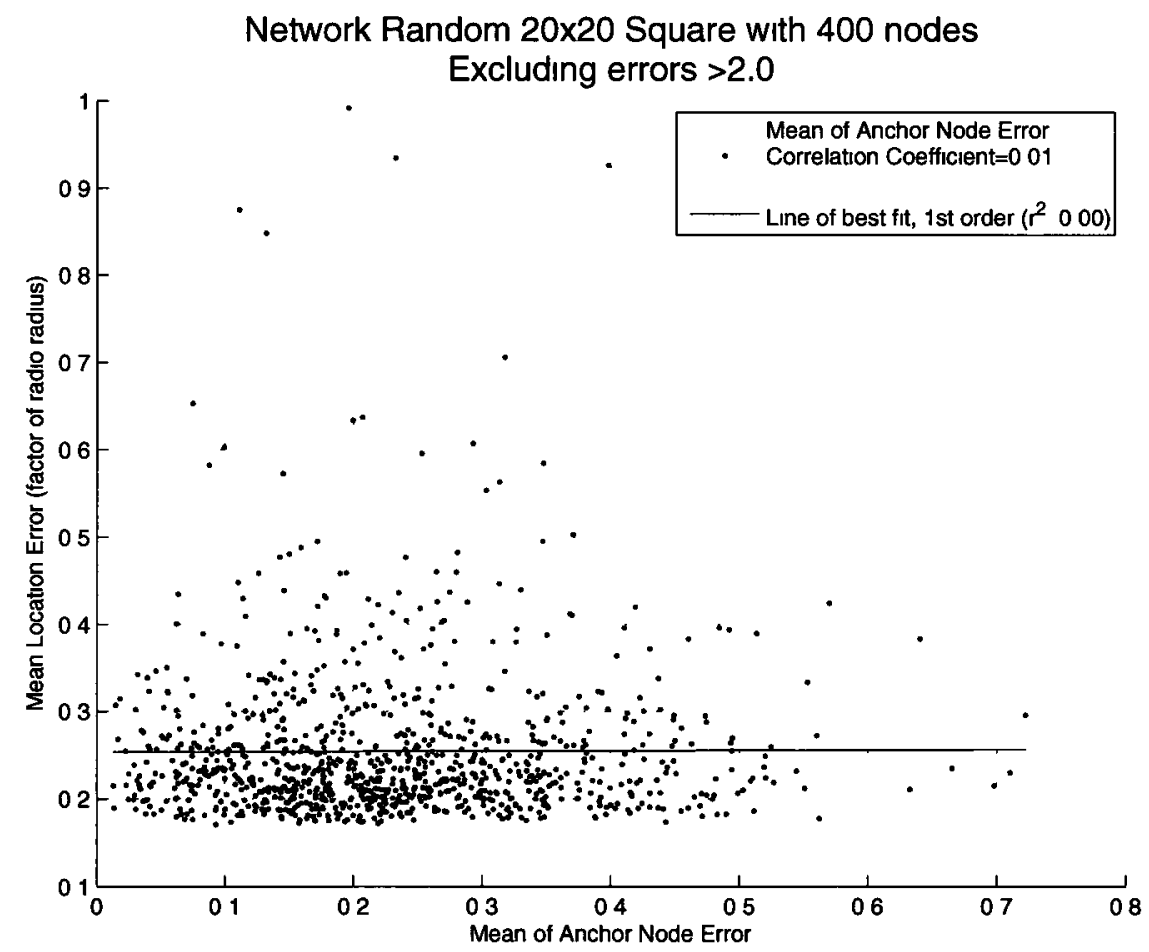

(b) Excluding outliers

Figure 4.4: Mean of anchor node error vs. location error 
of 0.02 . Higher order best fits also show no clear correlation.

\subsubsection{Network Area Coverage}

As suggested in related studies in Chapter 3, an optimal anchor placement is to have the anchors as far apart as possible, around the edges of the network. The rationale behind this hypothesis, in the context of this study, is that if a wider area of the network is covered by the anchor nodes, then the resulting calculated transformation will take into account more network variations.

One way to determine how close the anchors are to the perimeter of the network is to measure how far apart the anchors are from each other. The further apart they are, they closer they are to the edge and the more network area they cover. For statistical purposes, the sum of the distance between each pair of anchors is taken. I describe this as the sum of distances of the anchors instead of the perimeter of the triangle formed because this hypothesis investigates if the further apart the anchors are from each makes a difference. Further, the sum of distances scales for the case of more than three anchor nodes, whereas the meaning of perimeter will change the desired description. Figure 4.5 shows an example for both the three and four anchor node case. For the three node case, it is equal to the perimeter of the triangle. More generically, for more nodes, as shown here for four, it is the sum of all pairwise distances.

Figure 4.6a shows the sum of distances for each anchor set versus mean localization error for that anchor set. The plot shows that again there is low correlation between the distance between anchors and the location calculation. Further, the outliers are spread relatively evenly regardless of distance between anchor nodes, and thus this is not a good indicator of an outlier.

When the outliers are excluded, as shown in Figure 4.6b, a moderate level of 


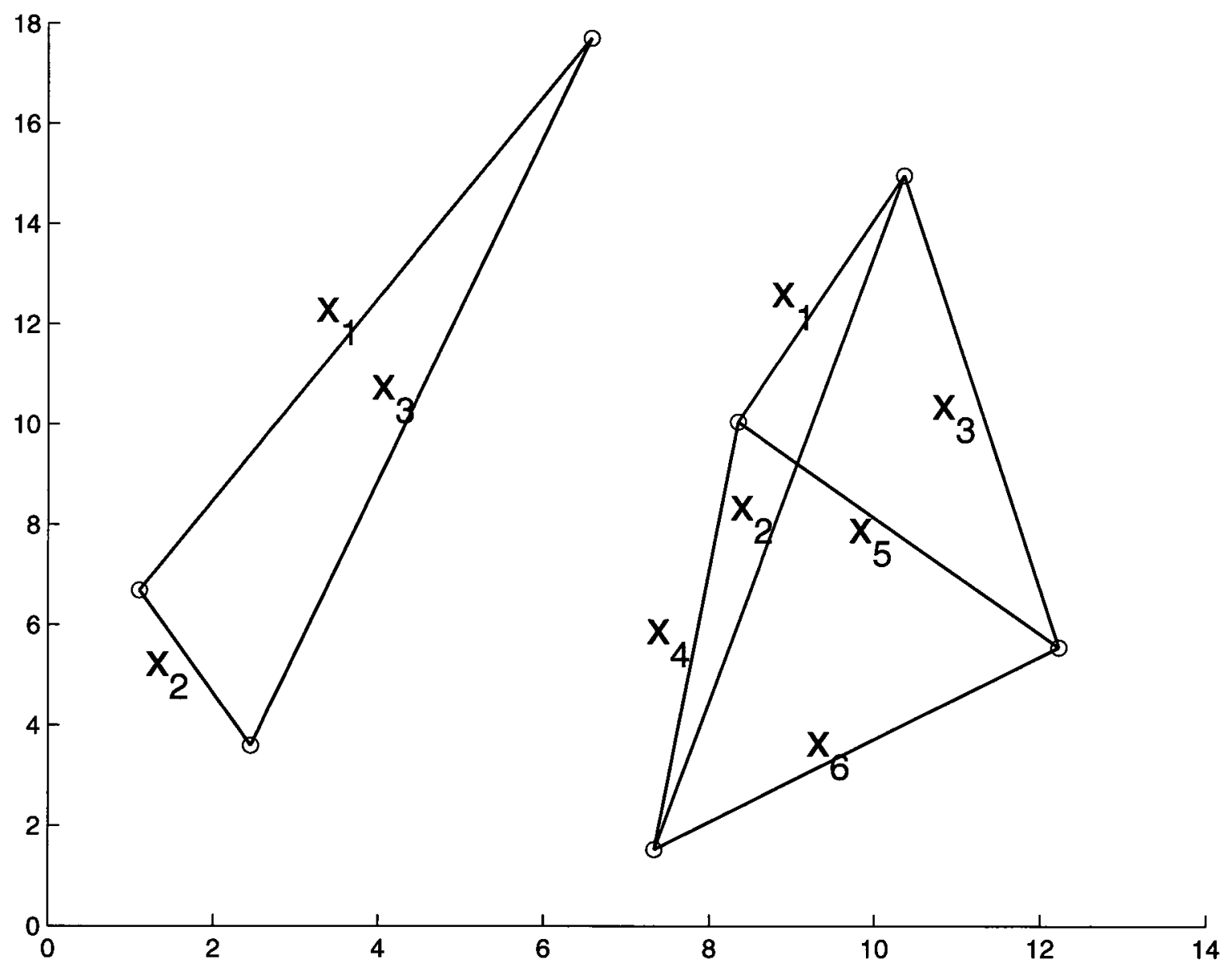

Figure 4.5: Sum of distances $=\sum_{\imath=0}^{n}\left\|x_{\imath}\right\|$ 


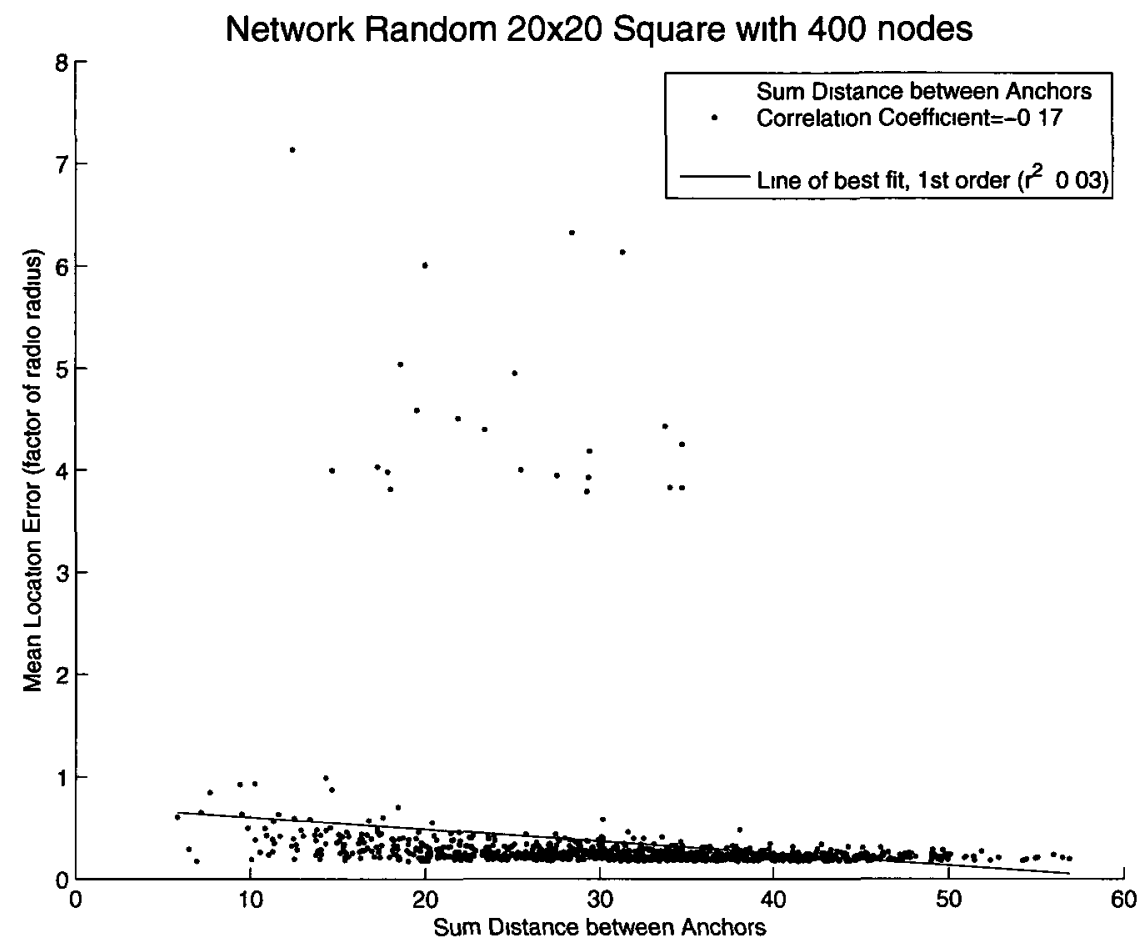

(a) All anchor sets

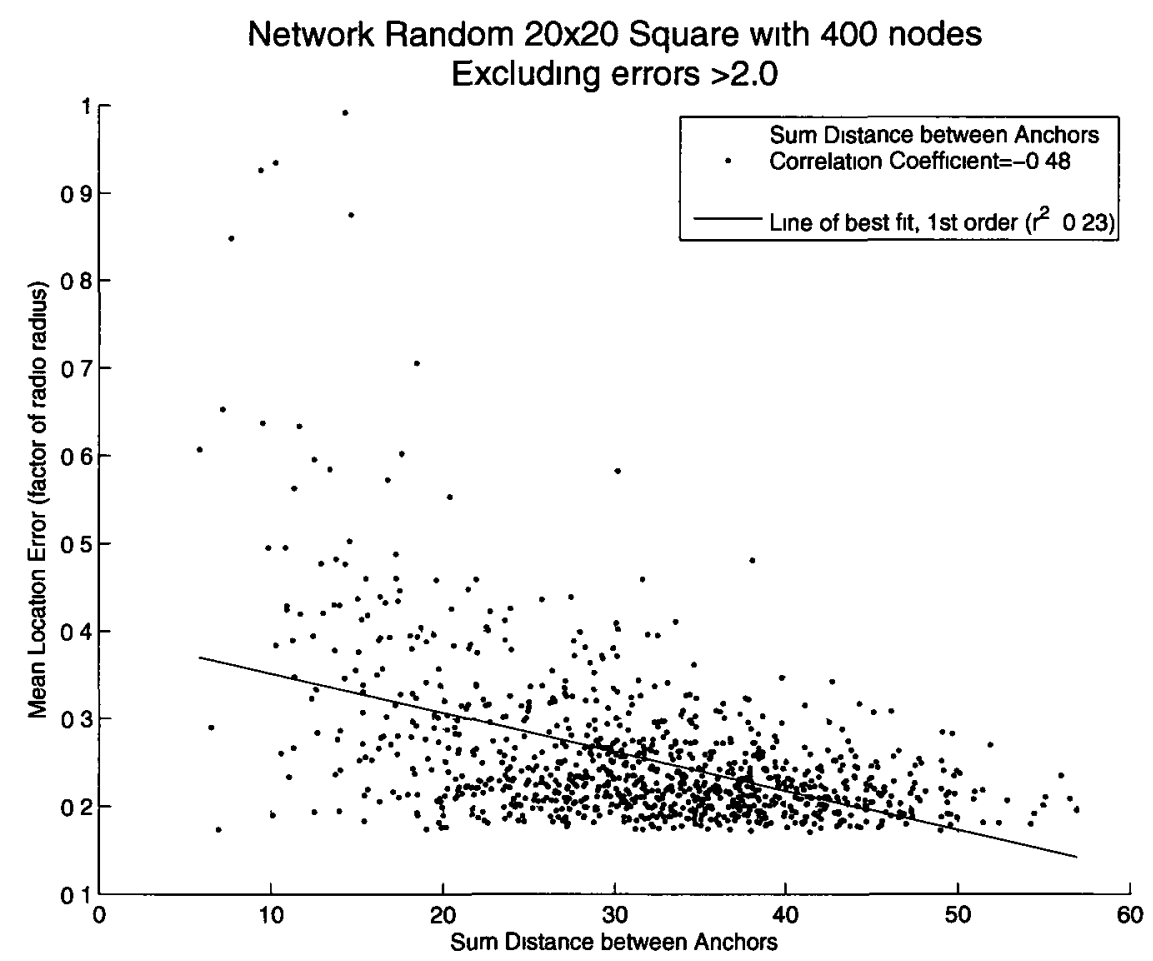

(b) Excluding outliers

Figure 4.6: Sum of distance between anchors vs. location error 


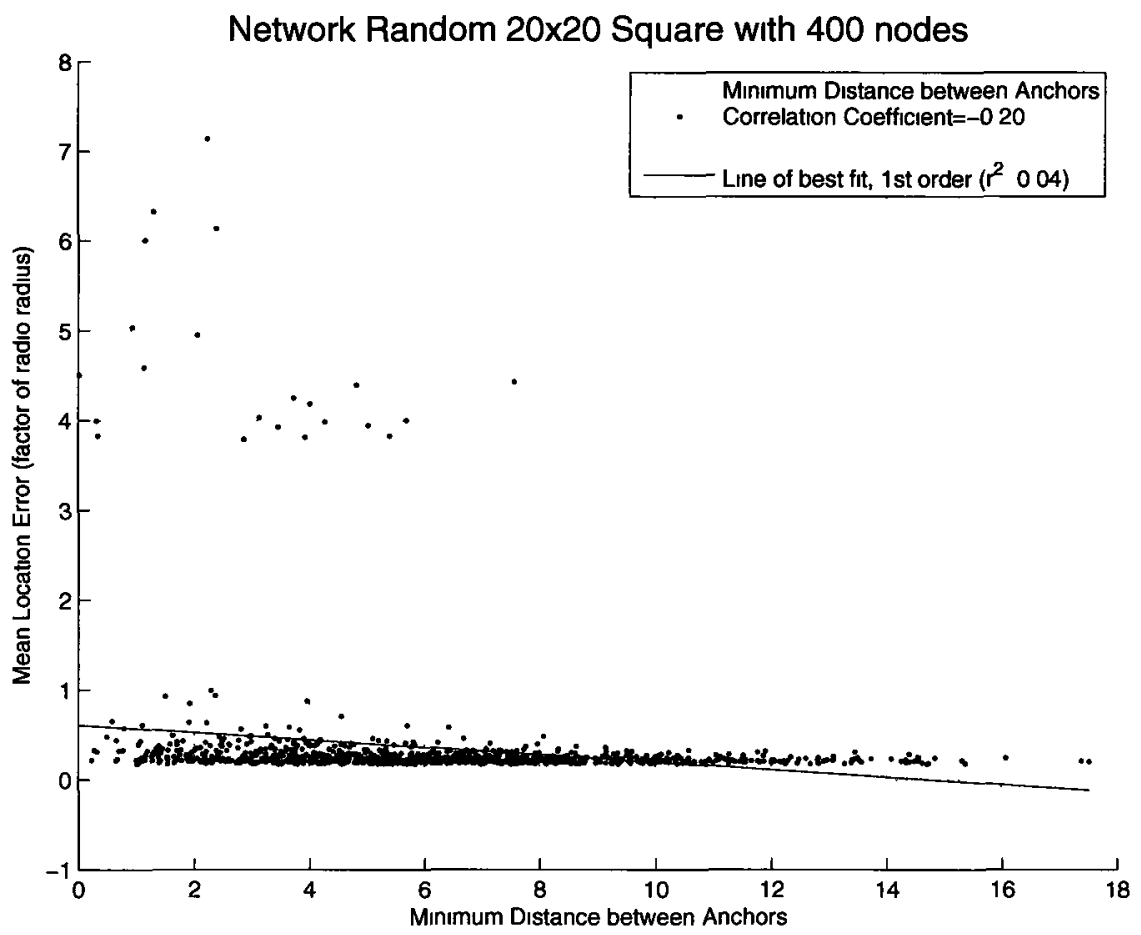

(a) All anchor sets

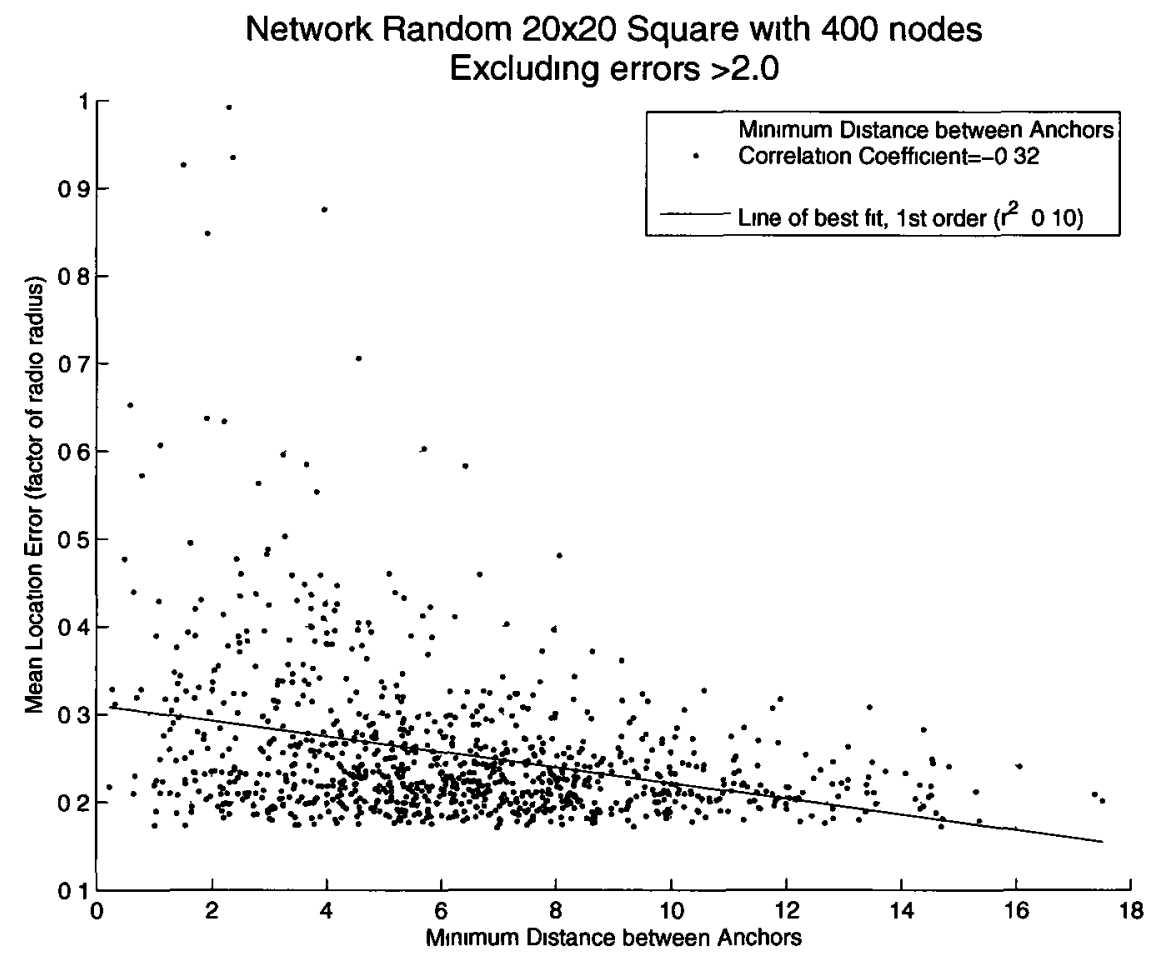

(b) Excluding outliers

Figure 4.7: Minimum distance between anchors vs. location error 
correlation is seen, with a much clearer line of best fit. The only moderate, 0.48 , correlation coefficient can be explained by the lower bound for location error. There is a virtually straight line across the bottom of the data indicating that, at any distance between anchors, the lower bound of mean location error can be reached. However, as the distance increases between the anchor nodes, the probability of getting a high location error decreases.

Even if the sum of distances of the anchors is high, it is possible for two anchors to be very close together and far from the third. Therefore, the minimum distance between a pair of anchors is shown in Figure 4.7a. Again, the correlation coefficient is low at 0.20 . However, it does appear that the outliers are slightly more likely to appear when the minimum distance between a pair of anchors is low. Further, when outliers are excluded, as shown in Figure $4.7 \mathrm{~b}$, the coefficient is 0.32 , slightly lower than that of the sum of the distances, implying that sum of the anchor distances is a better indicator of localization performance than minimum anchor distance.

\subsubsection{Anchor Node Triangle}

Continuing the trend of trying to show some increased correlation between the anchor

coverage and location error and based on speculative statements by Shang in [?, p.4] presented in Section 3.1, we propose and examine two additional metrics attempting to measure collinearity of the anchor nodes: the area and height of the anchor node triangle. For height, we calculate the height in each direction of the triangle, and use the shortest value. Both metrics can be extended to more anchor nodes. Figure 4.8 shows a simple example of calculating the area and minimum height of a triangle.

Figure 4.9 plots the area of the triangle formed by the three anchor nodes versus mean location error. This metric can also be extended to more anchor nodes, by using the area of the polygon formed by the anchor nodes. Figure 4.10 does the same for the 


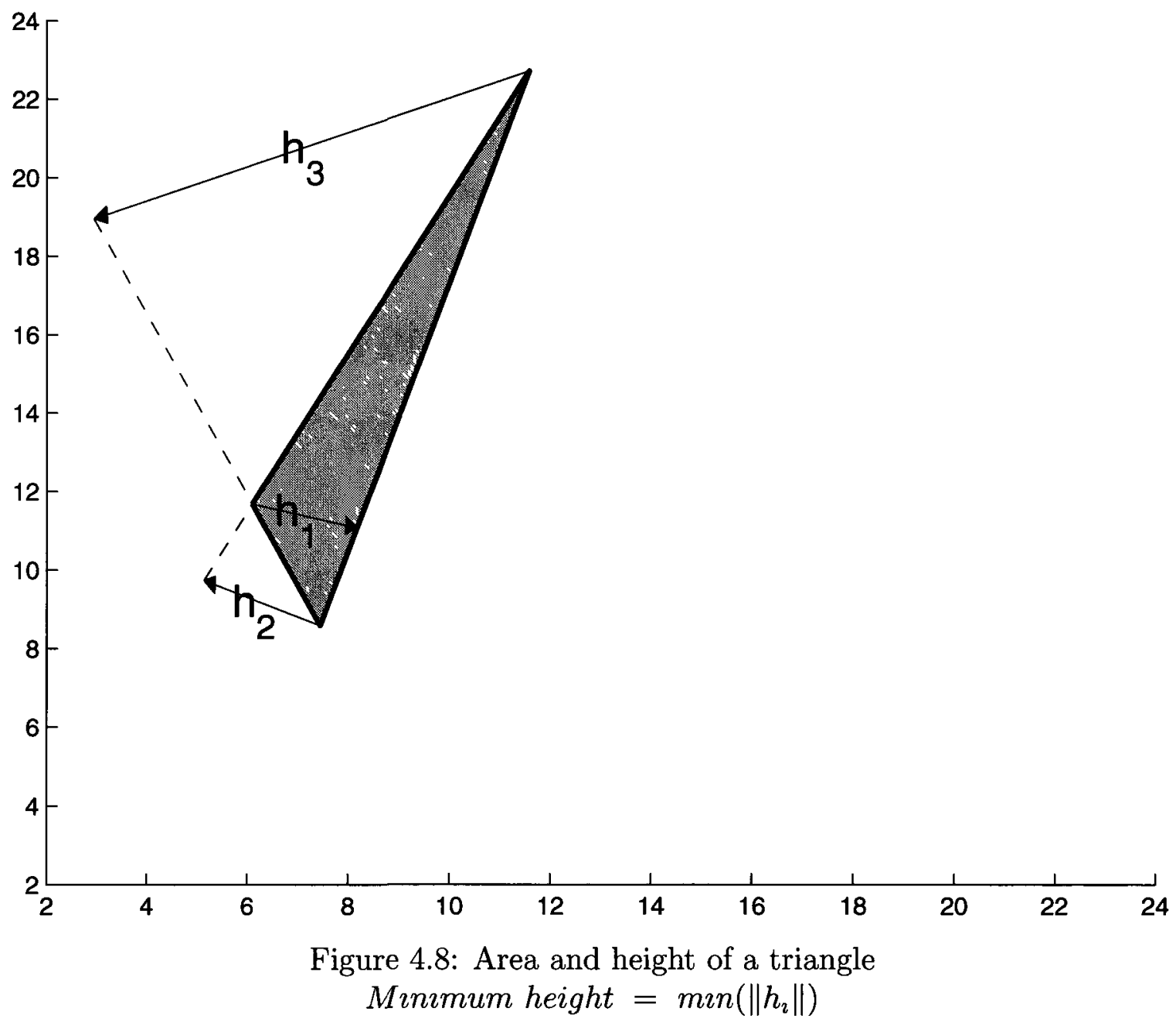


shortest triangle height. The first observation from the two plots is that the outlier cases are more tightly correlated to anchor area and height than any of the other metrics. The outlier points are all closer to the y-axis rather then being spread across the plot. This is explored further in Section 5.2. Secondly, after removing outliers, the anchor area has a slightly higher correlation coefficient than anchor height, indicating that anchor area is a slightly better predictor than anchor height.

\subsection{Best Anchor Node Placement}

Analyzing all the metrics discussed above, the best indicator of a good anchor placement is the sum of the distance between anchor nodes, once the outliers are excluded. This metric is best because it has the highest correlation to the mean location error. Avoiding the outlier case is discussed in detail in Chapter 5. Practically speaking, this means that network designers can choose between proposed anchor placements and use the measured distance between them to provide guidance about which placement will result in the least amount of error.

Figure 4.11 uses data from 20 networks, each occupying an area of $20 \times 20$ units with a radio range of 2.5 units. The networks have a density of one node per unit squared, but all nodes are randomly placed in each network. 5,000 anchor sets of three nodes each are chosen from the randomly placed nodes. The outliers are then removed from the data and the sum of distance between the anchors is plotted against the mean location error. The data is then grouped into two-unit intervals and a mean is calculated for each interval, to generate a histogram of the data. A horizontal bar through the mean of each interval displays the width of that histogram interval. Further, a $95 \%$ confidence interval of the mean value is shown as a vertical bar.

This plot shows us that as the sum of distances between the anchors approaches ten 


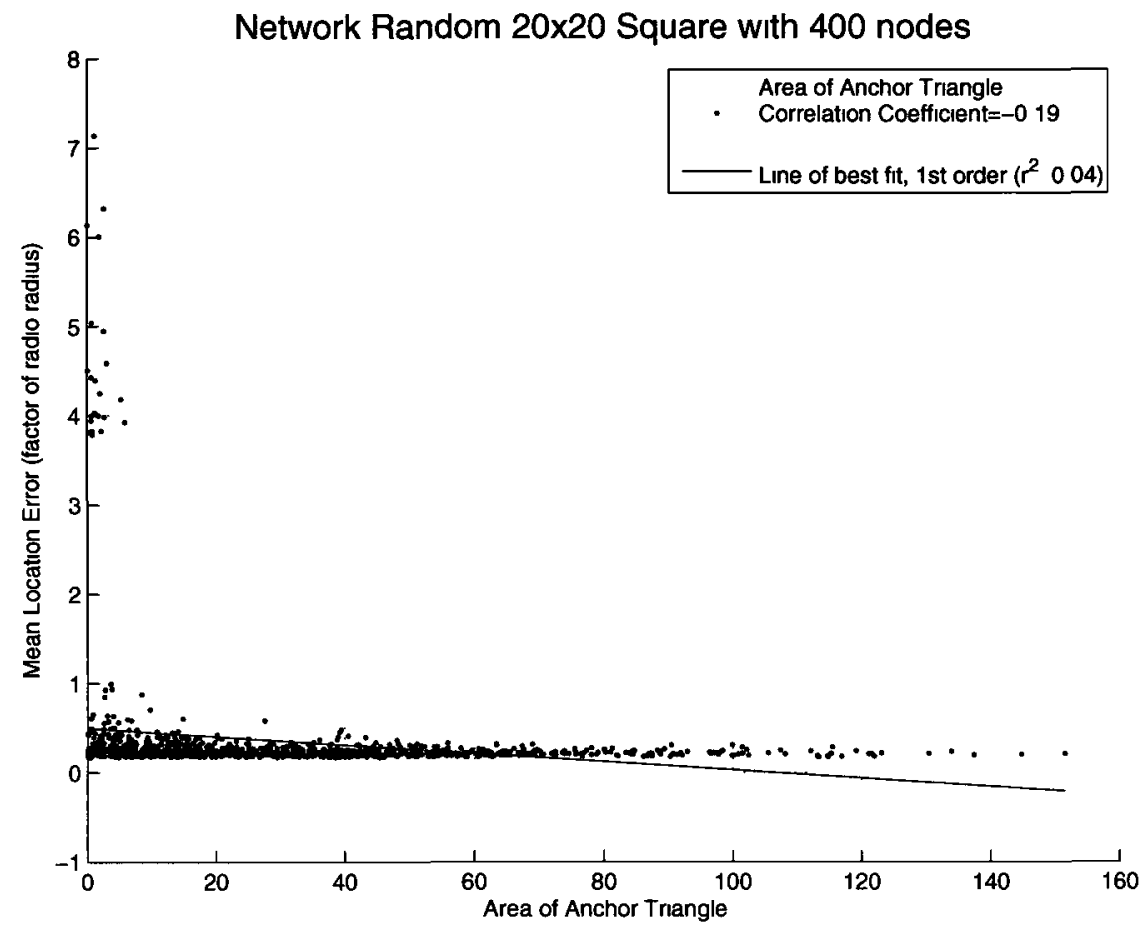

(a) All anchor sets

Network Random $20 \times 20$ Square with 400 nodes

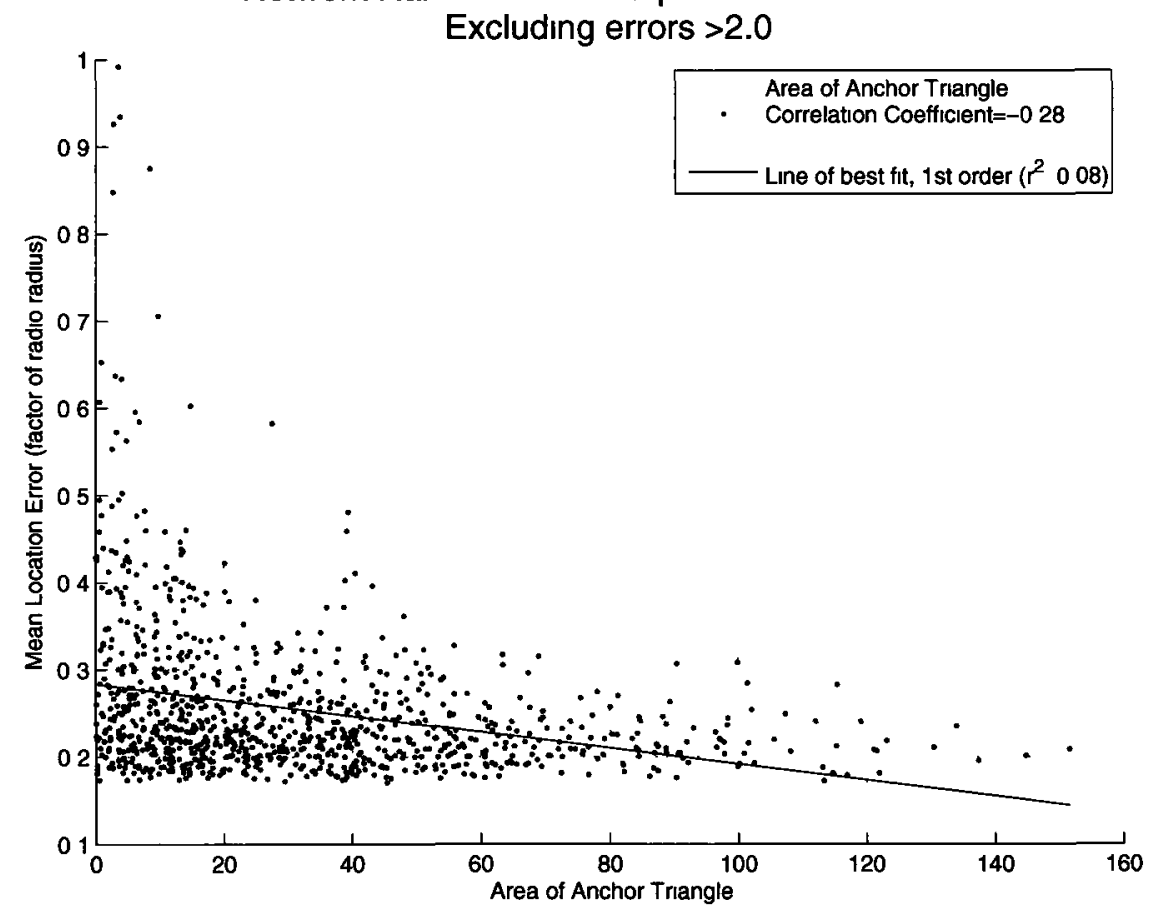

(b) Excluding outliers

Figure 4.9: Area of anchor triangle vs. location error 


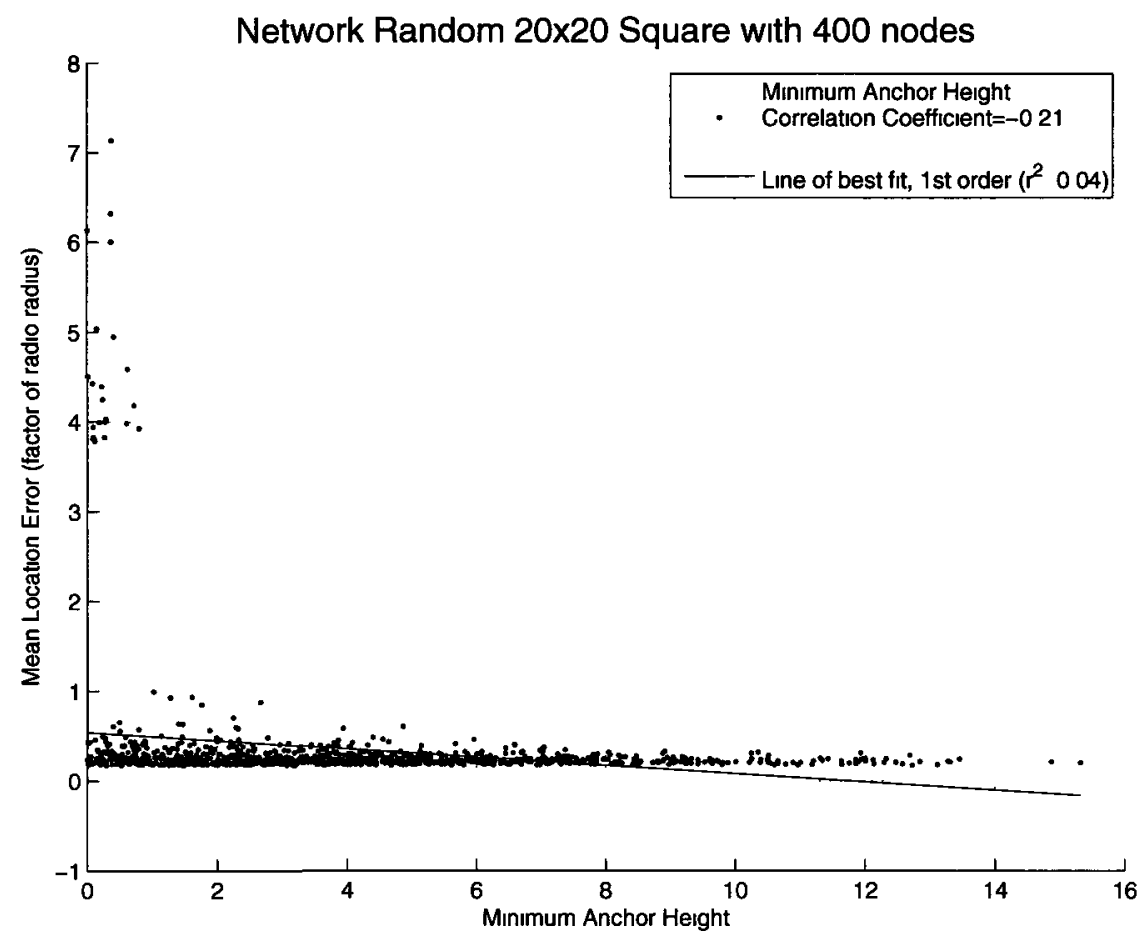

(a) All points

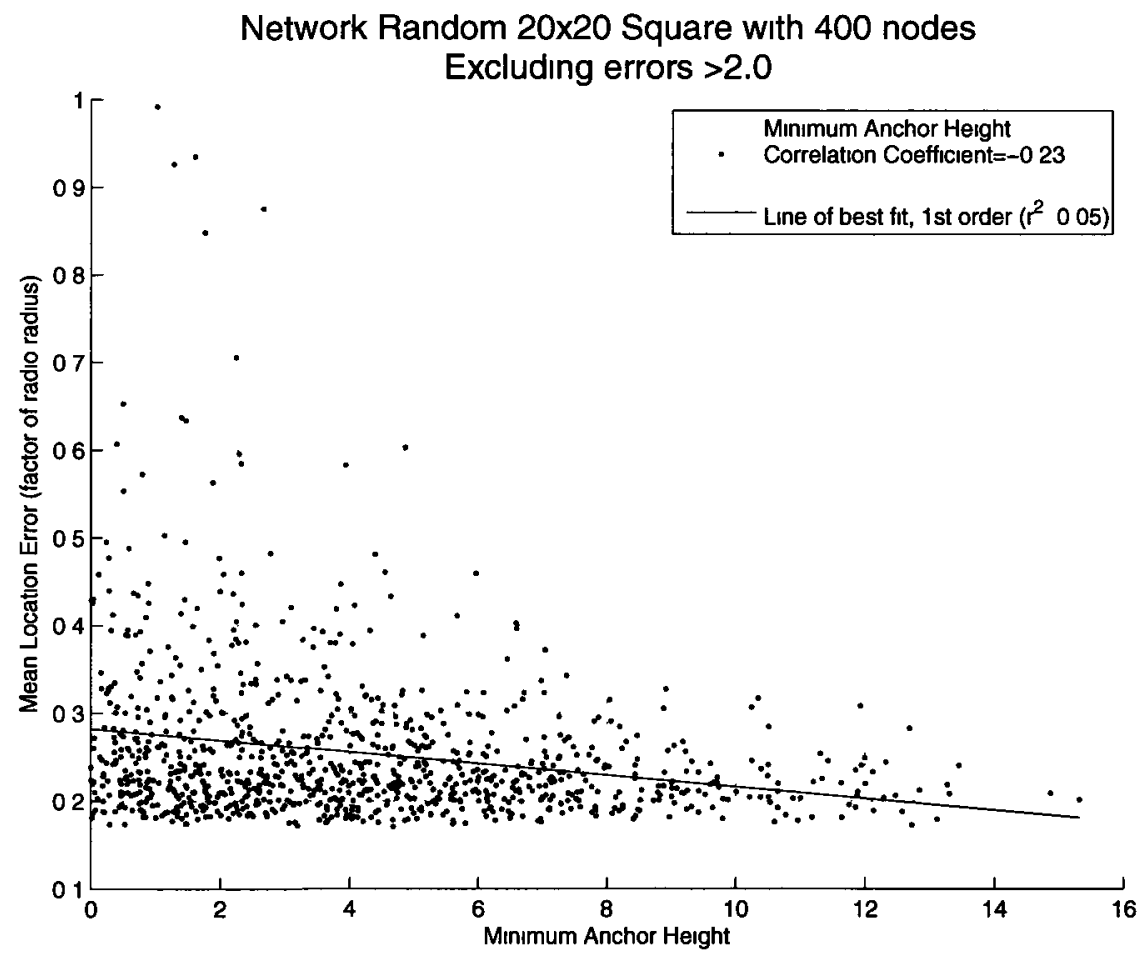

(b) Excluding outliers

Figure 4.10: Minimum height of anchor triangle vs. location error 


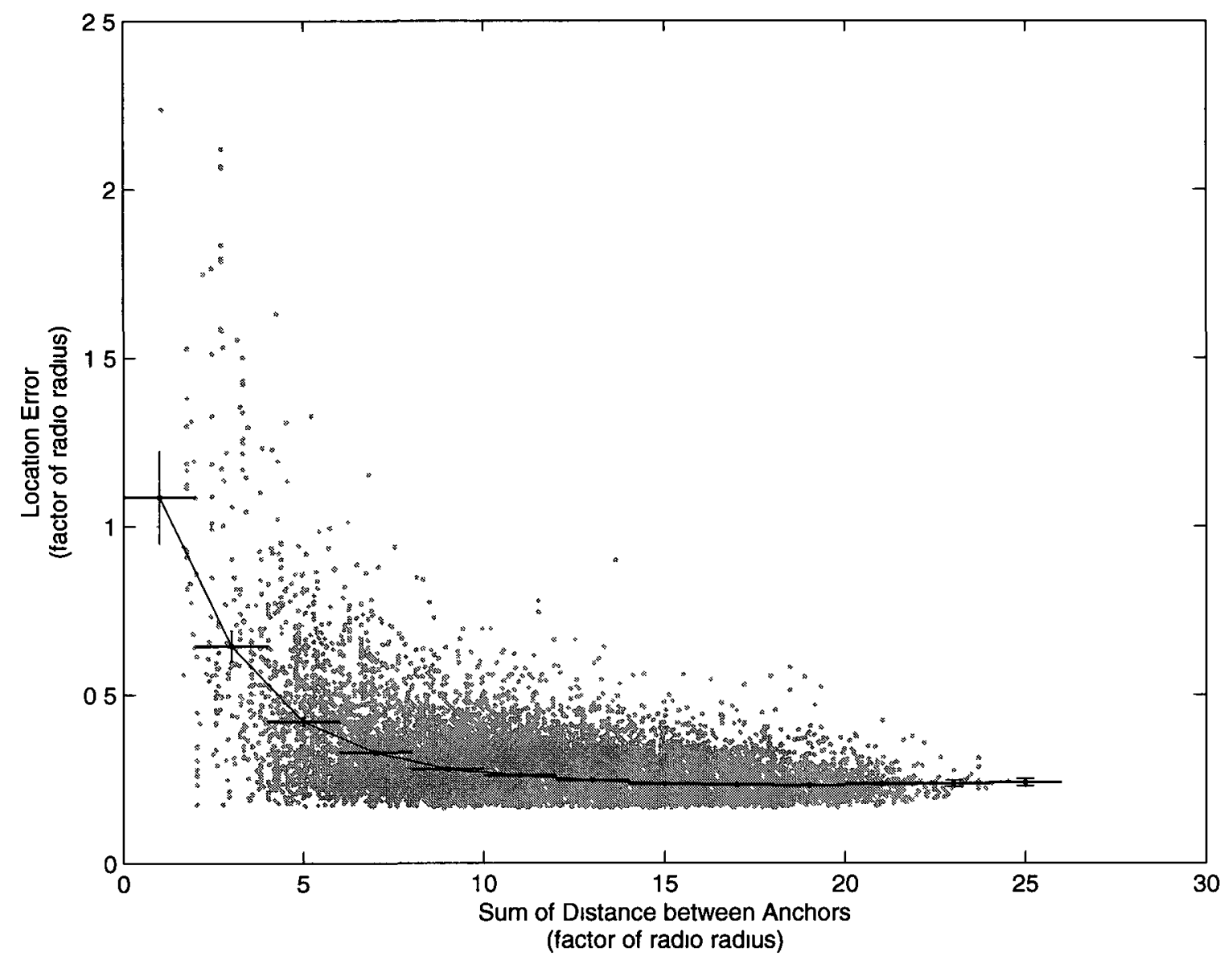

Figure 4.11: Sum of distance between anchors vs. location error for 20 random square 20-by-20 unit networks with 400 nodes, 5,000 anchor sets each, and a radio range of 2.5 units, excluding outliers

times the radio range, the differences in location error are statistically insignificant. Therefore, network designers can choose anchors nodes locations at their convenience, as long as they meet this criteria. The actual asymptote value depends on other factors, like network density and will be discussed further in Chapter 7 . 


\section{Chapter 5}

\section{Avoiding the Worst Anchor Node Placements}

As seen in the previous chapter, there are some anchor node placements that are significantly worse than the average case. In this chapter, we explore in more detail the cause of these outliers and more importantly, is this condition detectable a priori and hence preventable in the real world.

\subsection{Effects of Procrustes Analysis}

Figure 5.1a shows a network map of an example outlier anchor node placement for a random network. A line is drawn between the real location and the calculated location for each individual node. The length of this line is the location error. For purposes of comparison, Figure $5.1 \mathrm{~b}$ is a randomly chosen non-outlier case, where the node difference lines are short, representing low localization error. However, in the outlier case, the node difference lines criss-cross the network along a clear, single angle. This visual representation suggests the reflection component of the final linear transformation is to blame for the extremely poor, outlier results. 


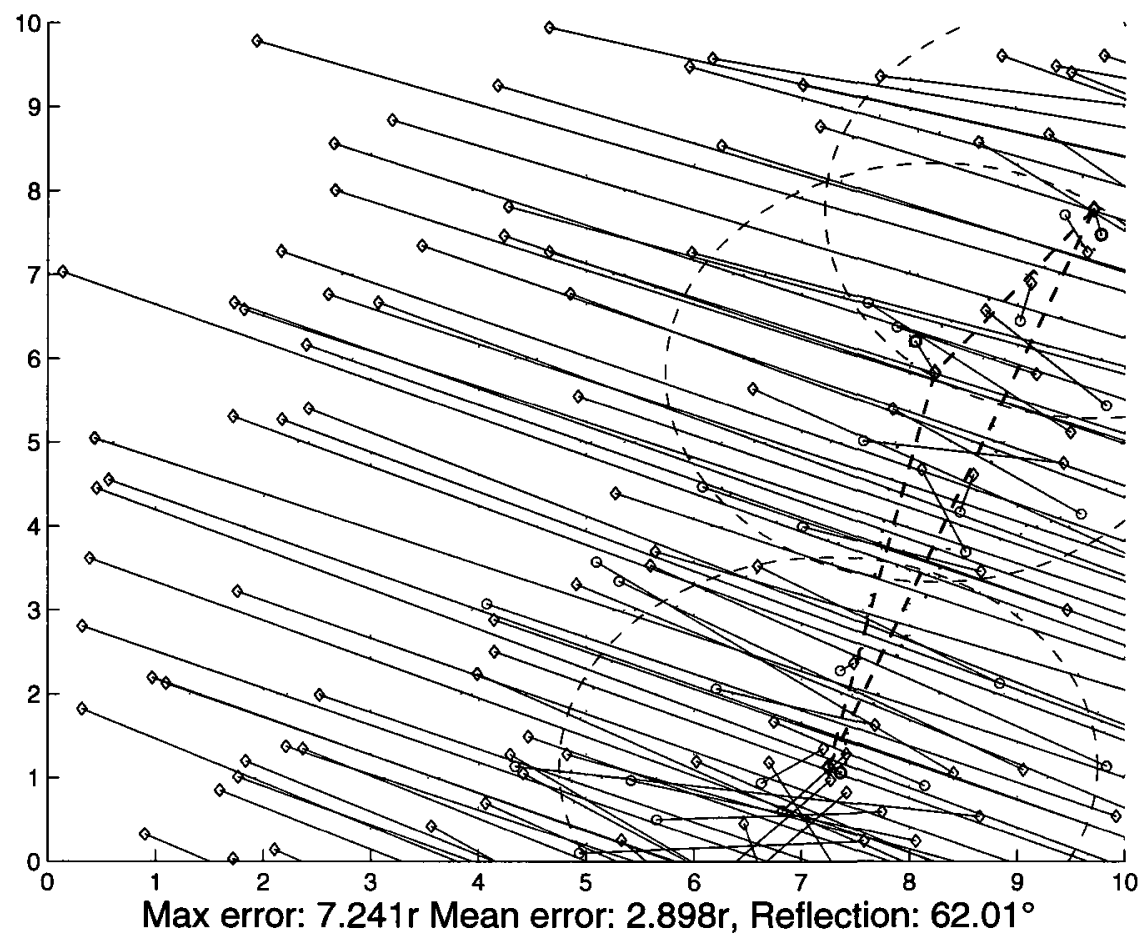

(a) An outlier network difference

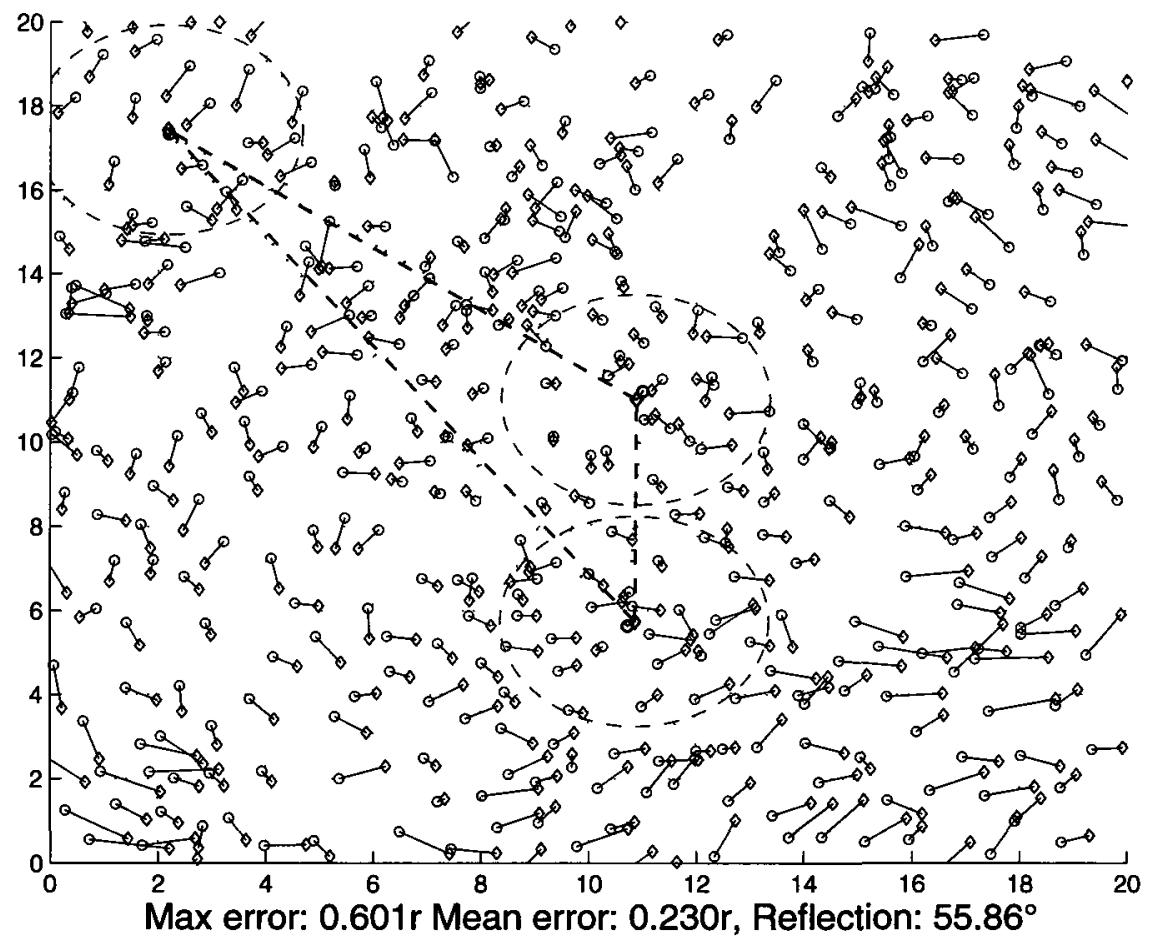

(b) A normal network difference

Figure 5.1: Network map of localization performance at each node 


\subsubsection{Transformation Reflection and Rotation}

Unfortunately, simply disabling the reflection component of the Procrustes transformation algorithm does not solve the problem. The output of the Procrustes algorithm is a linear transformation which includes a rotation or reflection matrix, as discussed in Section 4.2. If the determinant of that matrix is +1 , then the resulting transformation has a rotation, with an angle as in Equation 5.1.

$$
\operatorname{det}(T)=+1 \Rightarrow \text { Rotation with } T=\left[\begin{array}{cc}
\cos \theta & -\sin \theta \\
\sin \theta & \cos \theta
\end{array}\right]
$$

If the determinant is -1 , then the resulting transformation has a reflection component across a line at an angle as shown in Equation 5.2.

$$
\operatorname{det}(T)=-1 \Rightarrow \text { Reflection with } T=\left[\begin{array}{cc}
\cos 2 \theta & \sin 2 \theta \\
\sin 2 \theta & -\cos 2 \theta
\end{array}\right]
$$

Figure 5.2 shows the rotation and reflection distributions of two different networks, for a random set of anchor sets for each network. In both networks, and with consistency across others, the bulk of the data points have the same angle of either rotation or reflection, while the outliers have the opposite property with a wide variance in the angle. However, in different networks, it is not consistently either rotation or reflection that leads to poor localization results. Therefore, relying on the determinant of the transformation is not a sufficient indicator for a network designer knowing that an outlier case has been detected and that the localization results are essentially useless. 


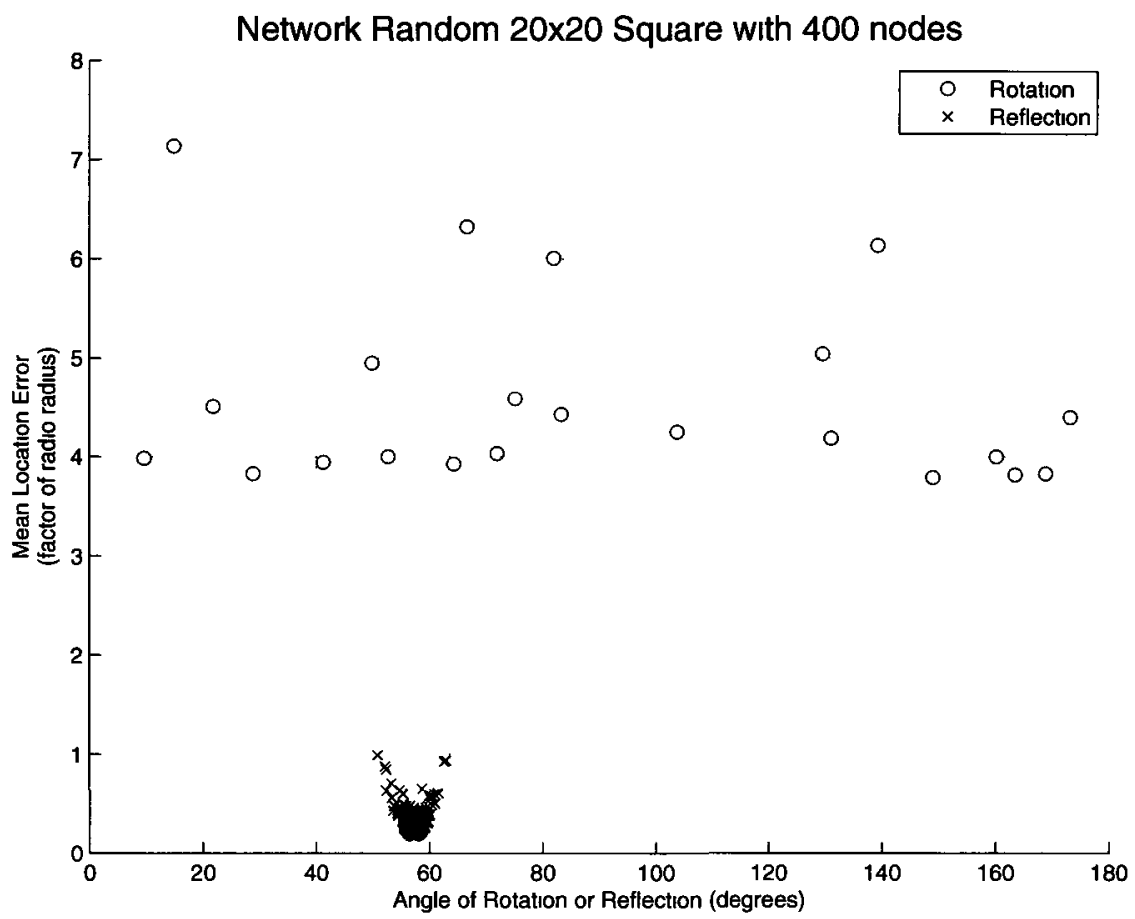

(a) A network where reflection is good

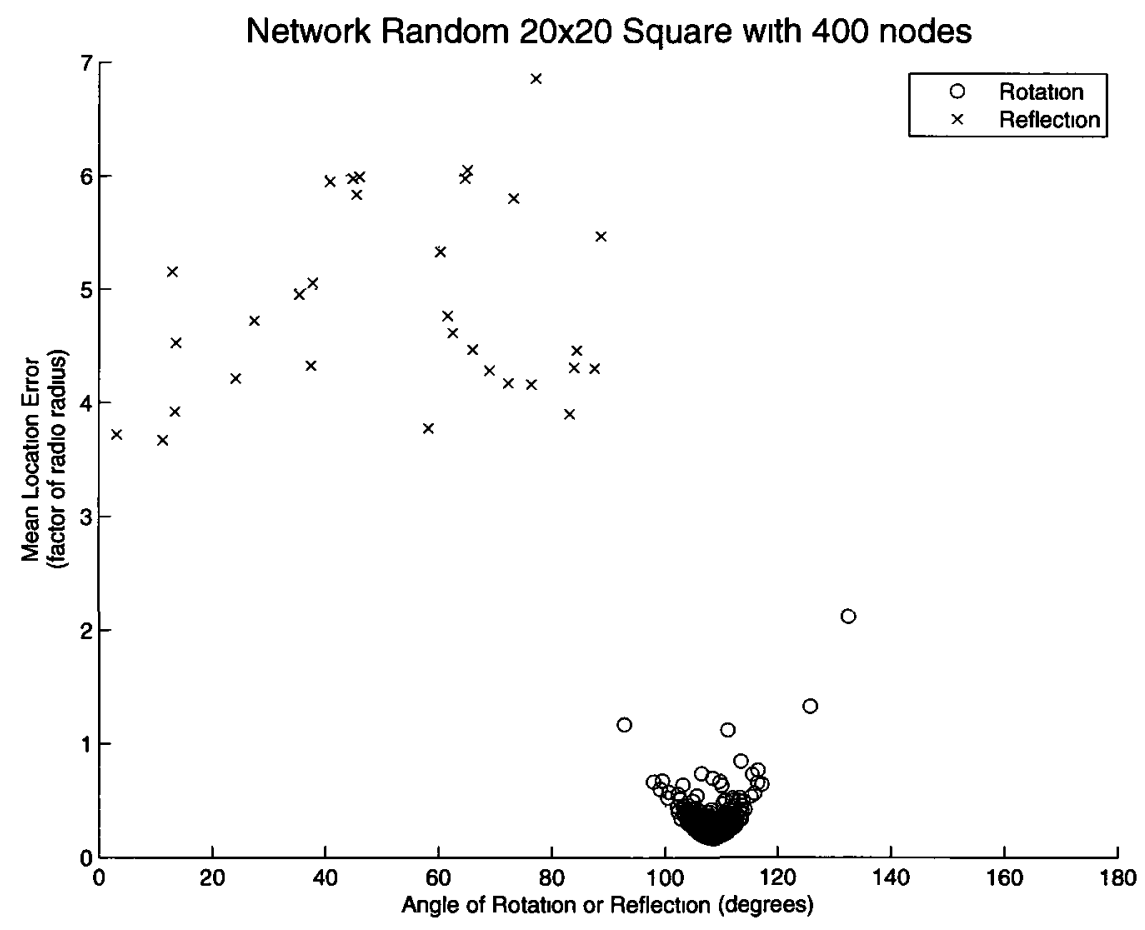

(b) A network where rotation is good

Figure 5.2: Rotation and reflection versus location error 


\subsubsection{Transformation Scaling and Translation}

For completeness, we explore the possibility that the scaling or translation component of the linear transformation is causing the issue. In Figure 5.3, the scale component, $b$, of the Procrustes analysis is plotted against the mean location error for the same two networks as shown above for reflection and rotation in Figure 5.2. While there is an apex of the data around a particular scalar value where the minimum localization error is seen, the outliers can also have this same scalar value. Therefore, the scaling component is not a good indicator of extremely poor localization performance.

Likewise, Figure 5.4 plots the horizontal, $x$, and vertical, $y$, translation components, $c$, against the mean location error. As with the other transformation components, there is concentration around a natural value in $x$ and $y$ that gives best localization performance. The fact that these $x$ and $y$ values overlap in Figure 5.4a is a coincidence. However, much like for the scaling factor, while outlier localization errors are the only values that occur away from this natural value, the outliers also occur around that value as well. Therefore, it is not possible to classify an anchor set as one of the outlier cases based on the translation component of the linear transformation it produces.

\subsubsection{Procrustes Dissimilarity}

The Procrustes algorithm itself provides a measure of the dissimilarity. Specifically, it is the minimized value of sum of squared errors [?]. As shown in Figure 5.5, it is not a good indicator of the transformation as it pertains to the entire network. This is because the Procrustes algorithm is only performed on the anchor nodes themselves and for those nodes themselves, the transformation is good. 


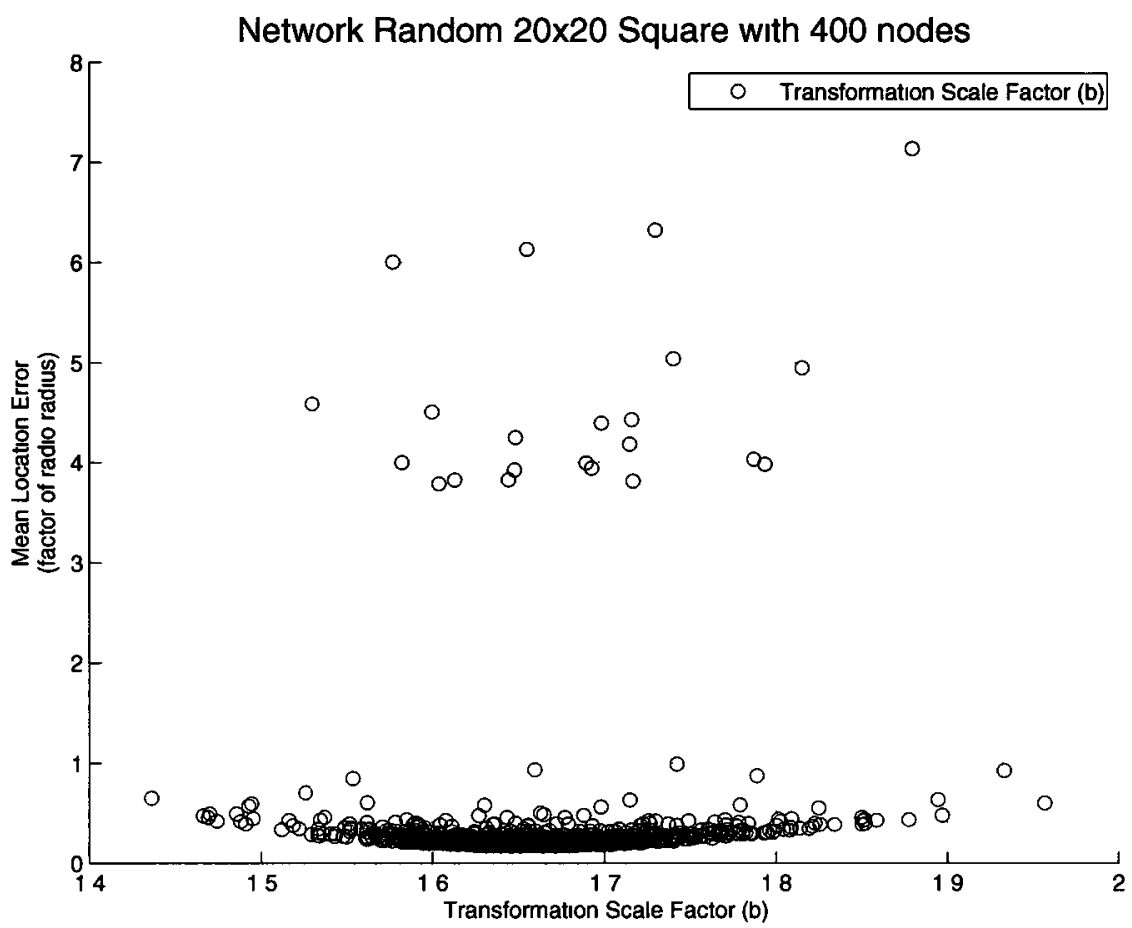

(a) Corresponding to a network where reflection is good

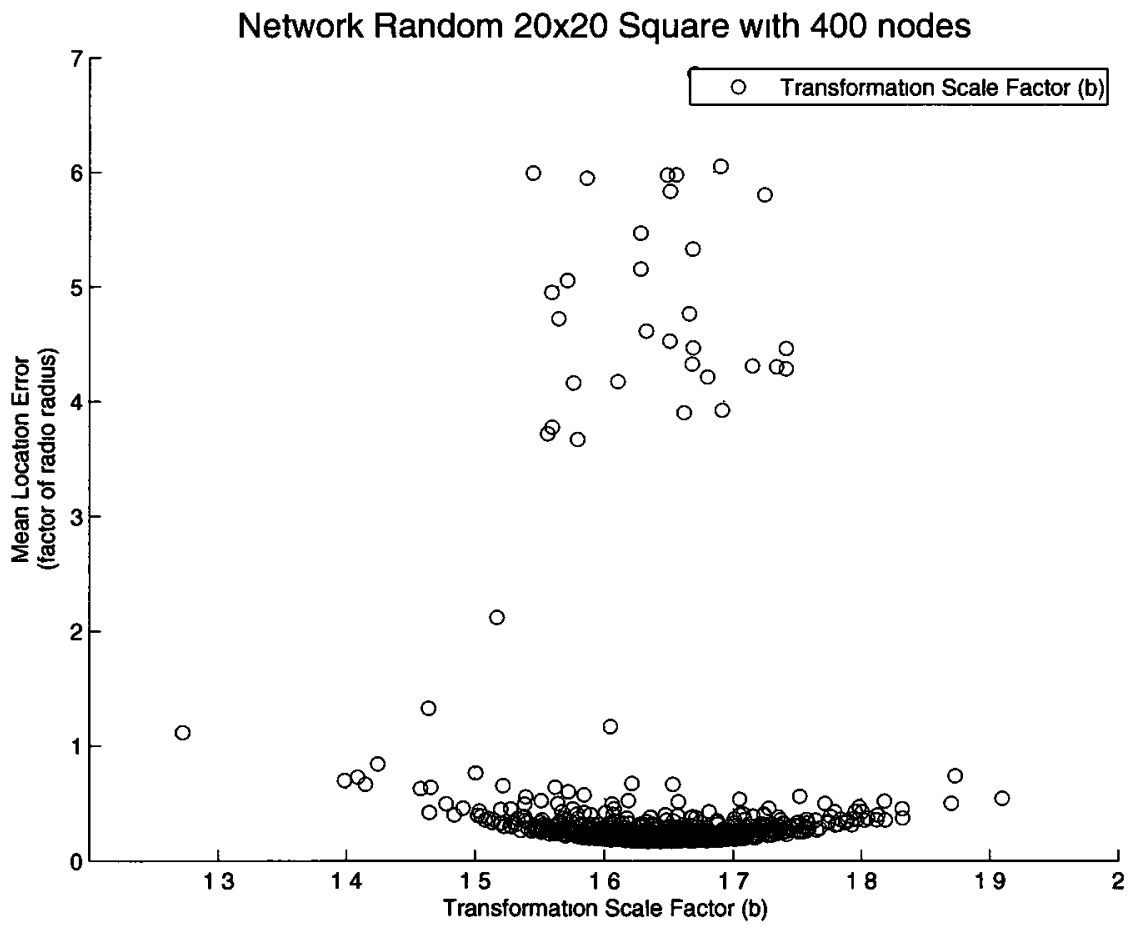

(b) Corresponding to a network where rotation is good

Figure 5.3: Transformation scalar component versus location error 


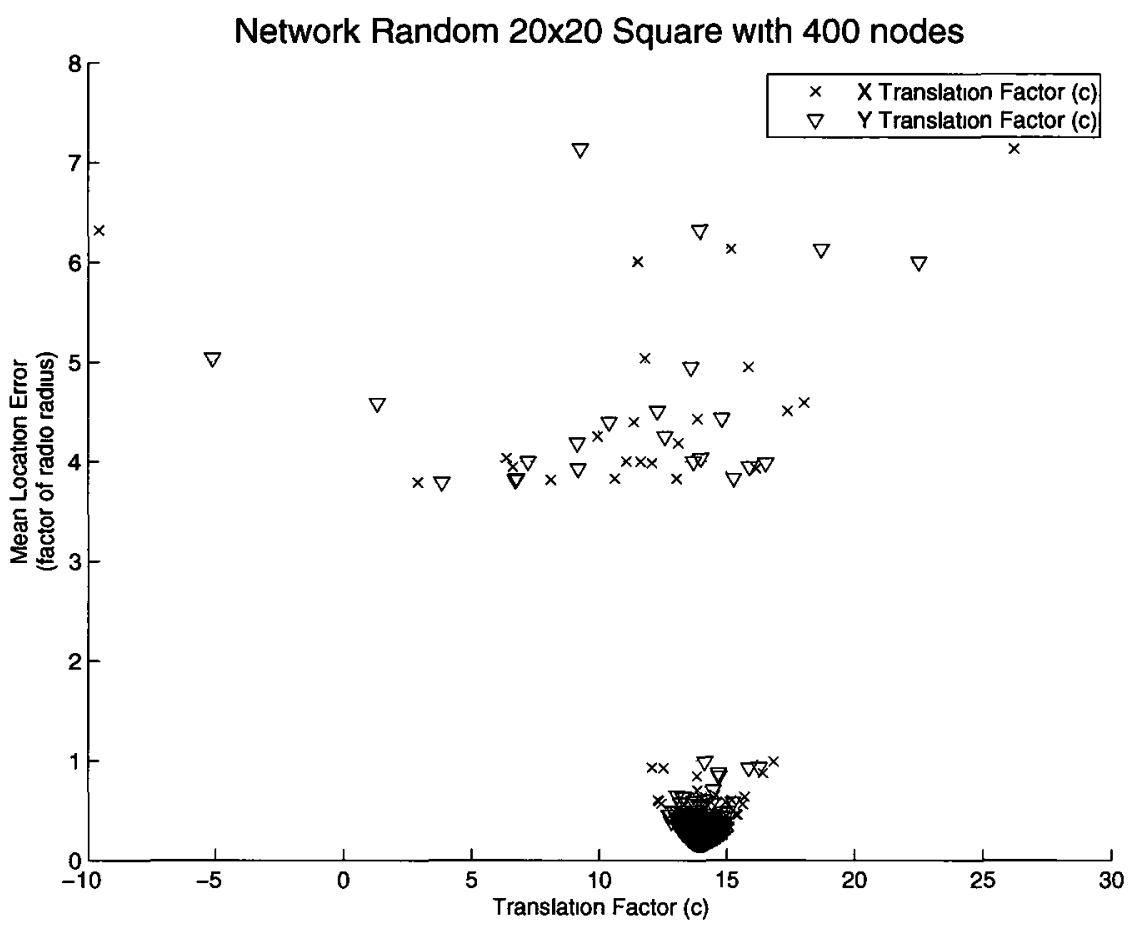

(a) Corresponding to a network where reflection is good

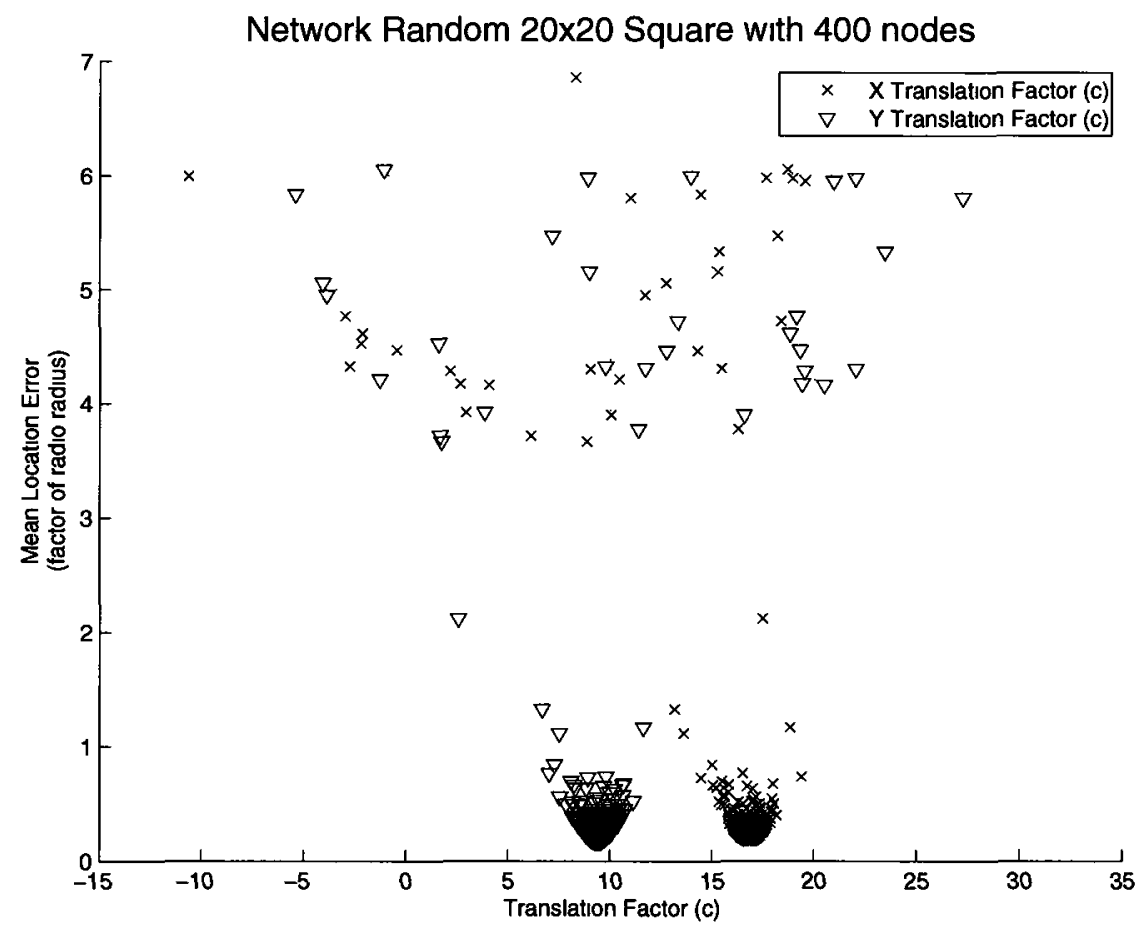

(b) Corresponding to a network where rotation is good

Figure 5.4: Transformation translation component versus location error 


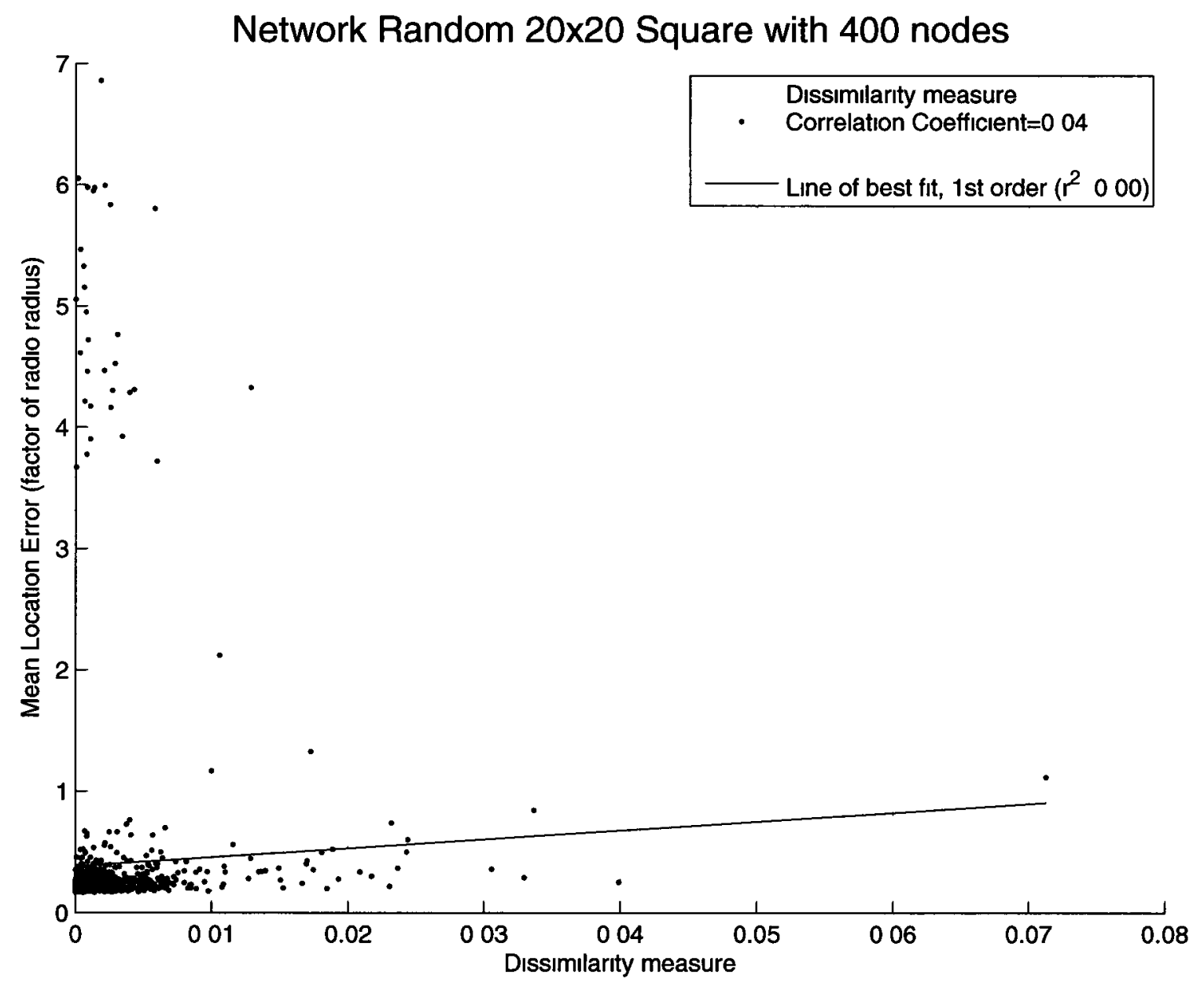

Figure 5.5: Procrustes dissimilarity measure 


\subsection{Outlier Indicators}

As shown above, there unfortunately is no direct indicator that a particular anchor set generates a transformation with an incorrect angle. Therefore, network designers must avoid all anchor sets that could potentially generate such an angle. As demonstrated in Section 4.4.3, the area or height of the triangle formed by the anchor nodes is a good indicator of the possibility of a poor transformation angle. These two metrics are explored in Figures 5.7 and 5.6, respectively. This time, the $\mathrm{x}$-axis is a log scale to better show the outlier cases. Also, the area and height have been adjusted as a factor of radius. Further, $95 \%$ confidence intervals are shown for the mean localization error of each $0.1 \mathrm{r}$ increment of both area and height. A solid horizontal line is shown throw each mean value, indicating the width of the increment, since it can be difficult to visualize on a log scale. The horizontal dashed line indicates the cutoff for what are considered outliers, based on the large gap in data points. The vertical dashed line indicates the first interval in which there are no outliers. As the area and height increase, the mean localization error decreases, as does the confidence interval of that mean.

Upon comparing the two plots, it is clear that the minimum height metric is far more stable when it comes to predicting outliers than the area metric. This is based on the observation that the mean localization error monotonically decreases with increased minimum height, while the mean localization error relative to area is far more erratic. Further, there are some outlier cases after the first interval of triangle area that has no outliers. A triangle with small area might provide a false-positive indication of an outlier. After careful thought, this result is not unexpected since height of a triangle geometrically exposes collinearity better than simply area of a triangle.

Based on the minimum height statistics, we can now give network designers a 


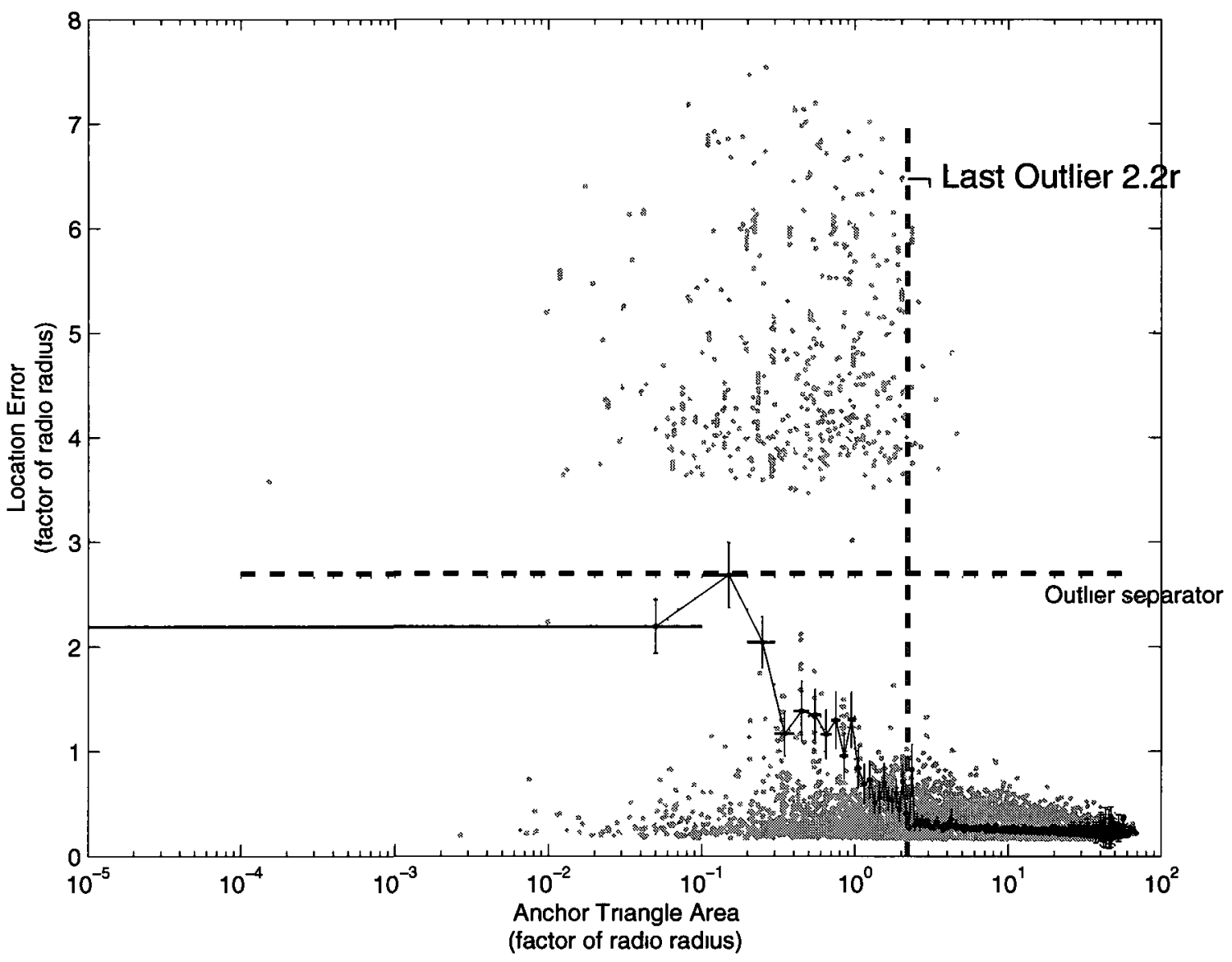

Figure 5.6: Anchor triangle areas, with confidence intervals grouped in intervals of $0.1 \mathrm{r}$ 


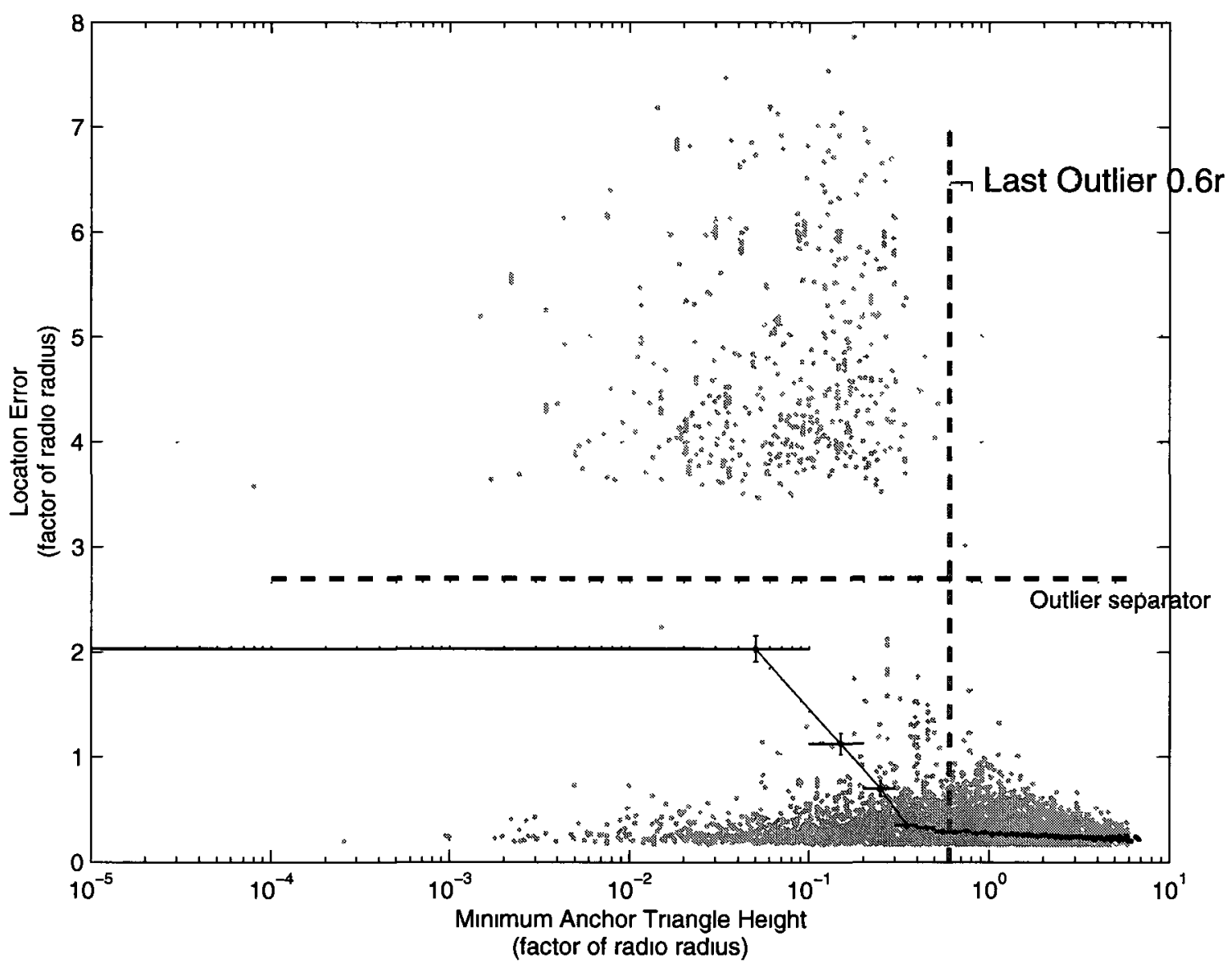

Figure 5.7: Minimum anchor triangle heights, with confidence intervals grouped in intervals of $0.1 \mathrm{r}$ 
metric of how collinear is too collinear for a set of anchor nodes. The raw statistics indicate that if the triangle formed by the anchor nodes has a minimum height greater that 0.6 times the radio range, then there is very low probability of getting an extreme outlier case were the calculated locations are actually reflected or rotated across the network. However, also in the data shown, there are some near-outlier cases beyond this point. While these cases are not of the extreme nature discussed, it may be worthwhile to give a margin of error here to avoid these cases as well. Therefore, we assert that the triangle formed by the anchor nodes should have a minimum height equal to the radio range of the network. 


\section{Chapter 6}

\section{Effects of Network Topology}

\subsection{Node Distance from the Anchors}

Of great assistance to network designers would be to know in which regions of the network to expect poor localization performance. With this information, and if the anchor placement is constrained, they could either avoid placing nodes in the expected poor area, or take into account the higher expected localization errors in the analysis of the data.

Figure 6.1 shows a different view of errors within two sample networks in attempt to find a correlation between sensor node location and each node's location error, especially based on relative location to the anchor nodes. The plot, instead of showing a line representing the localization errors as in other figures presented in this study, the error at each node is used to interpolate a grid of location errors throughout the area of the network. A contour plot, based on that interpolated grid, is then shown in the figure. The anchor nodes are shown, with their radio range shown as a dashed circle.

Figure 6.1 shows two random networks, with the same anchor node placements. In general, the region around the anchor nodes themselves has better localization 
performance, shown with darker color. However, there is quite a large variability in errors throughout the network. This simple example demonstrates that, unfortunately, no significant geographic correlation between location error and anchor node placement can be ascertained. As shown in Section 4.4.1, the localization error has almost no correlation with the density of nodes around anchor nodes or the error of the anchor nodes themselves. Therefore, the lack of correlation of any sensor node's location relative to the anchor nodes is not surprising.

However, there are likely other factors, such as network density, algorithm parameters to the underlying localization protocol of CCA-MAP, or yet undiscovered factors, that may be masking any it may be possible, correlation to the location of a sensor node relative to the anchor nodes. Therefore, with future study, it may be possible to remove the effect of these other factors to reveal a pattern based on anchor placement.

\subsection{Applicability to Varying Network Topologies}

So far, we have examined anchor node placement for a continuous square network, with randomly placed nodes. However, in the real world, networks are not alway so simple. There are often regions in the network where it is not possible to put nodes, due to physical barriers like lake, buildings or access to property. In this section, we look at how the results thus far apply to C-shaped and long, narrow pipeline network topologies.

\subsubsection{C-Shape Network Topology}

A C-shape network consists of a relatively square region with an empty area on one side, as shown in Figure 6.2. In terms of anchor placement requirements, we do not 


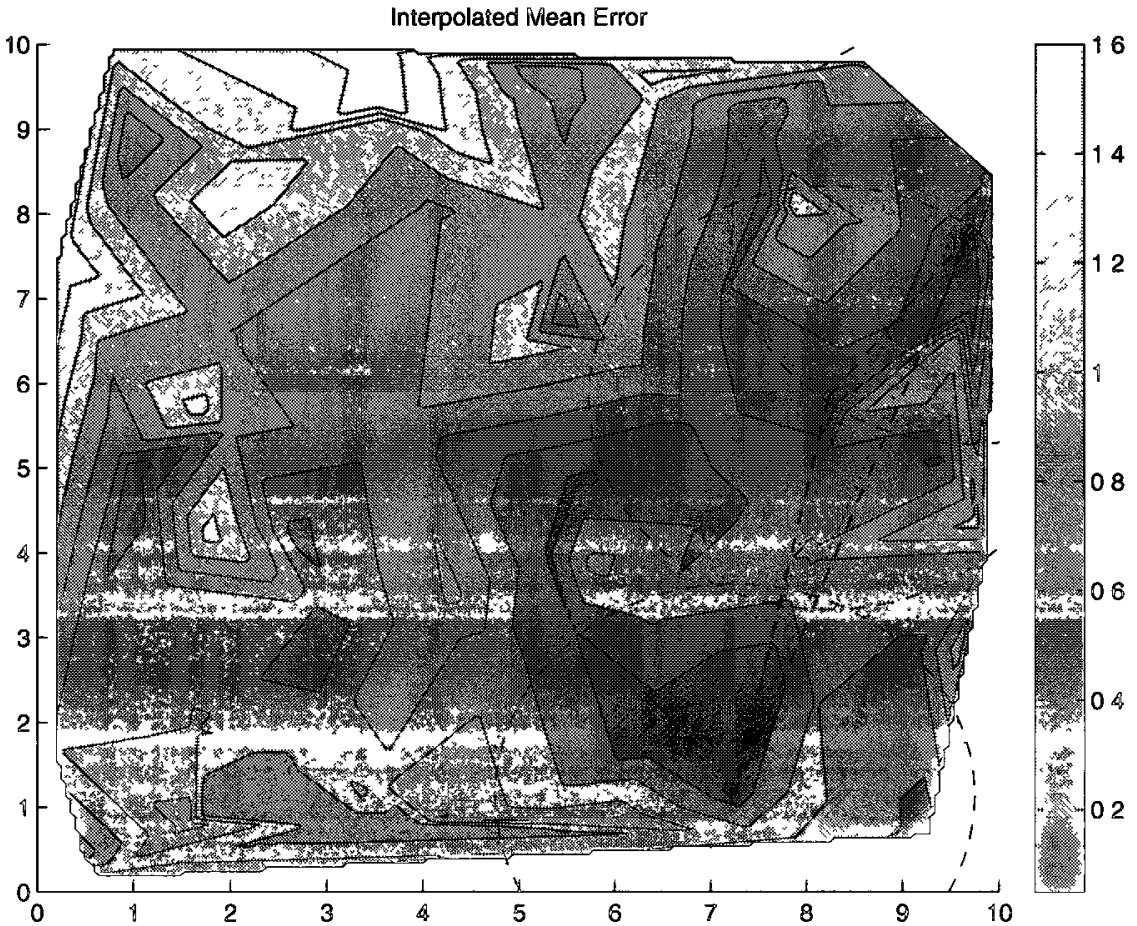

(a) Network $A$

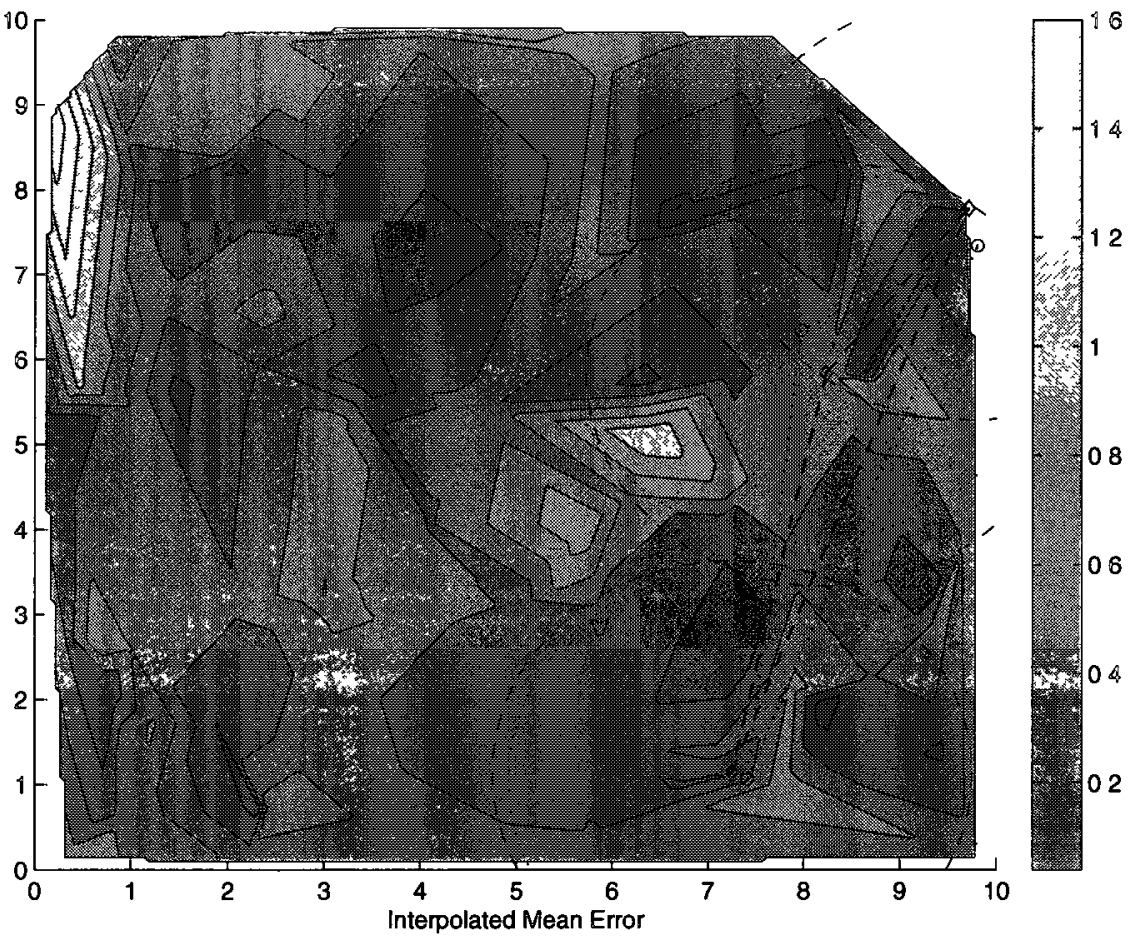

(b) Network B

Figure 6.1: Localization error as a contour plot 


\section{Shape Random $20 \times 20$ \\ Radıo Range: 25}

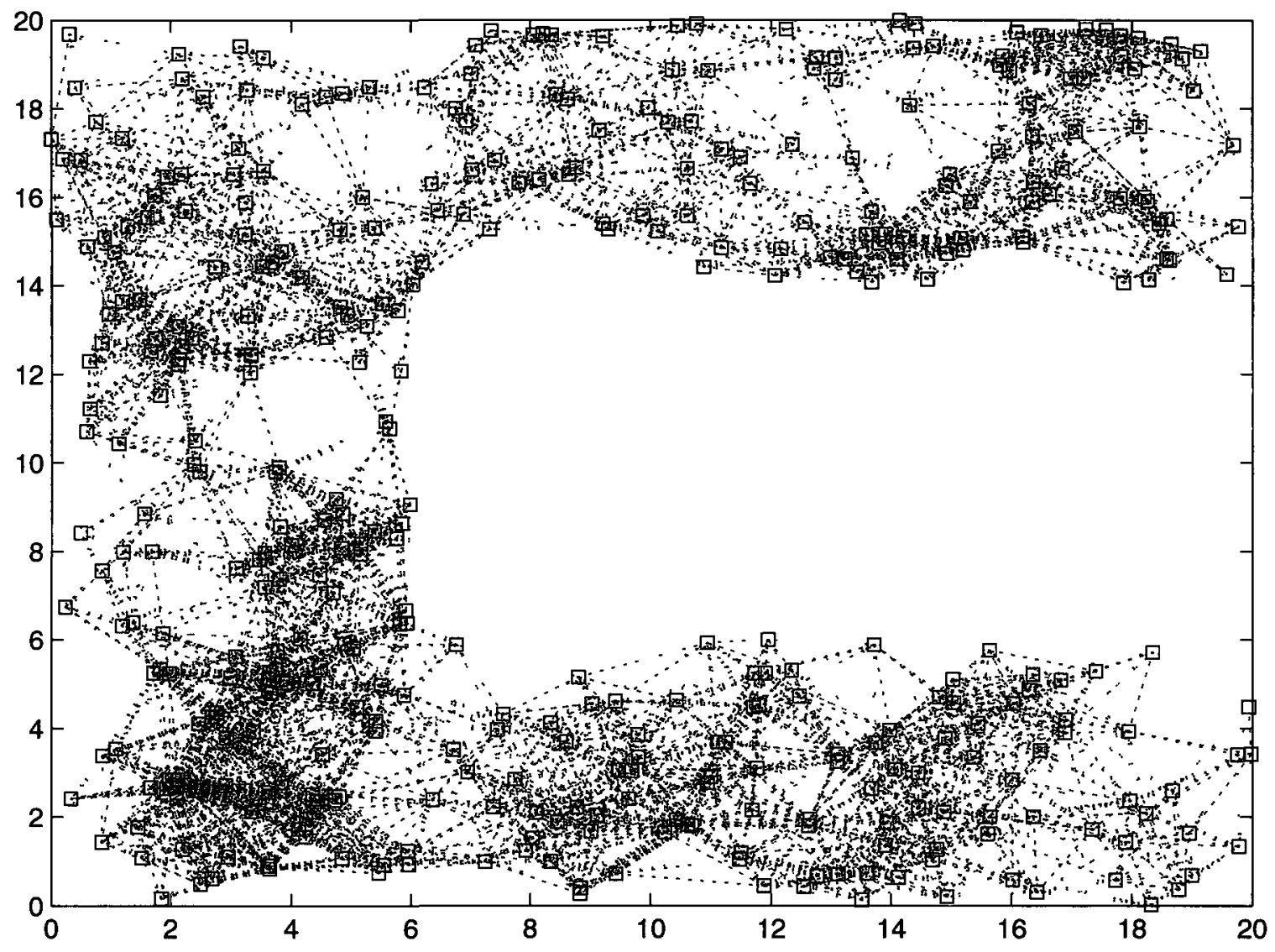

Figure 6.2: Example of C-shape network topology

expect any difference between the recommendations presented for square networks.

To show that there is no difference between C-shape and square network topologies, 10 random C-shape networks with 5,000 anchor sets each were simulated in the same manner as the square networks. Figure $6.3 \mathrm{a}$ shows the location error relative to the sum of the distances between the anchors, as in Section 4.5. As expected, the mean location error flattens out as the sum of distances between anchor nodes reaches about 10 times the radio range as was the case with the square network. There is a slight increase in the floor of the mean location error as compared with the 
square network, but this has to do with the performance of the CCA algorithm in the presence of the empty region of nodes in the network. The increase in mean location error at the end of the plot, and specifically the increase in the confidence interval, is caused by the small sample size in the random selection of anchor set with a very high distance between nodes.

Similarly, the same criteria for eliminating the outlier localization results also holds for C-shape topology as it did for square in Section 5.2. Figure 6.3b shows that as long as the minimum height of the triangle formed by the anchors nodes is greater than the radio range, then the outlier case can be avoided, as is the case for a square network.

\subsubsection{Pipeline Network Topology}

In some applications, the network region has very little depth to it, such as when monitoring a gas pipeline or along a highway or railroad line. The extreme case is where there is a single node placed along a straight axis. As we have shown, this is the worst possible scenario, as it is the most likely way to cause the outlier condition for localization. In that case, it is worthwhile to explore the possibility of other localization techniques, such as GPS at each node, or recording the location as the nodes are placed.

However, when there is a bit of depth to the network, as shown in Figure 6.4, there is still the possibility of good localization results. To demonstrate the importance of having an anchor node triangle height of at least the radio range, Figures 6.5 and 6.7 show mean location error for four different networks, with increasing network depth, but the same length.

As before, we attempt to distinguish the outlier case with a plot of the sum of distance between anchors. Figure 6.5 shows that as the network has a larger depth, 


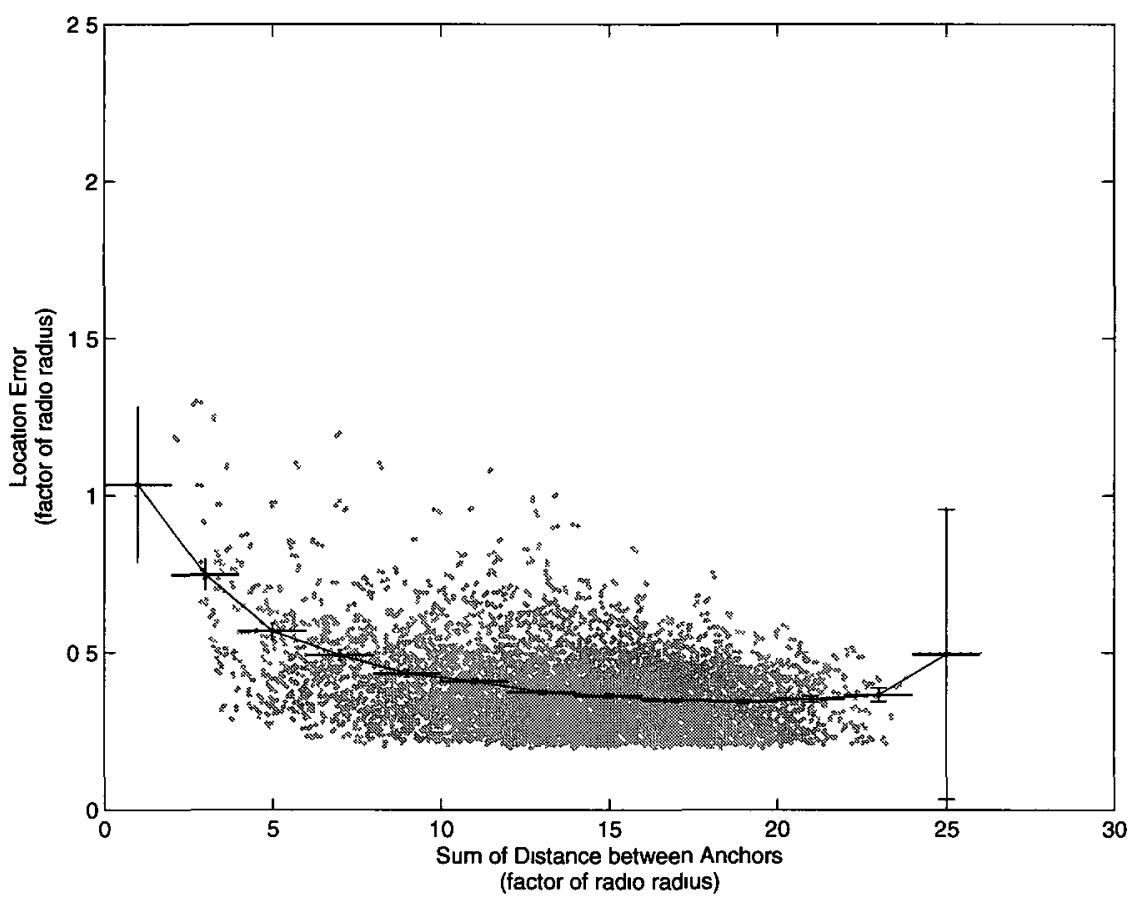

(a) Sum of distance between anchors vs mean location error, excluding outliers

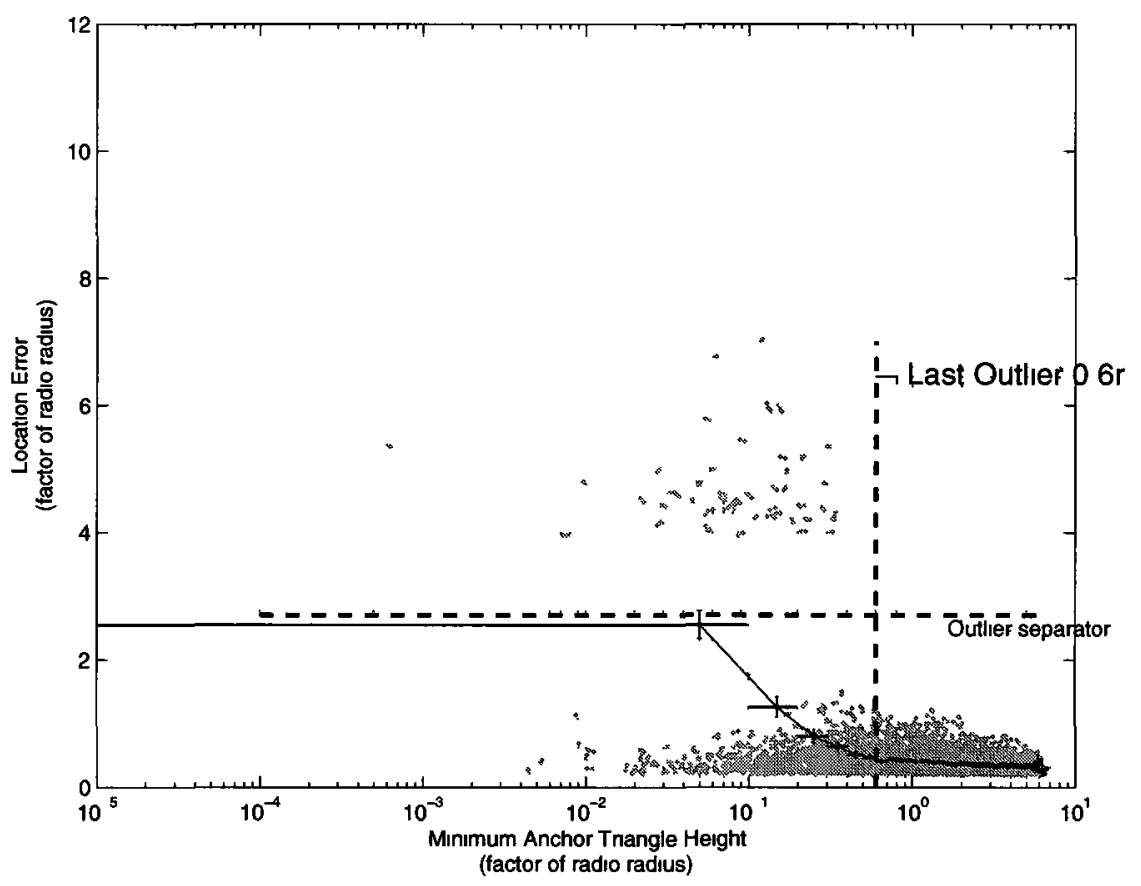

(b) Minımum height of anchor triangle vs mean location error

Figure 63 : Anchor metrics for 10 random C-shape 20-by-20 unit networks with 400 nodes, 5,000 anchor sets each, and a radio range of 25 units 


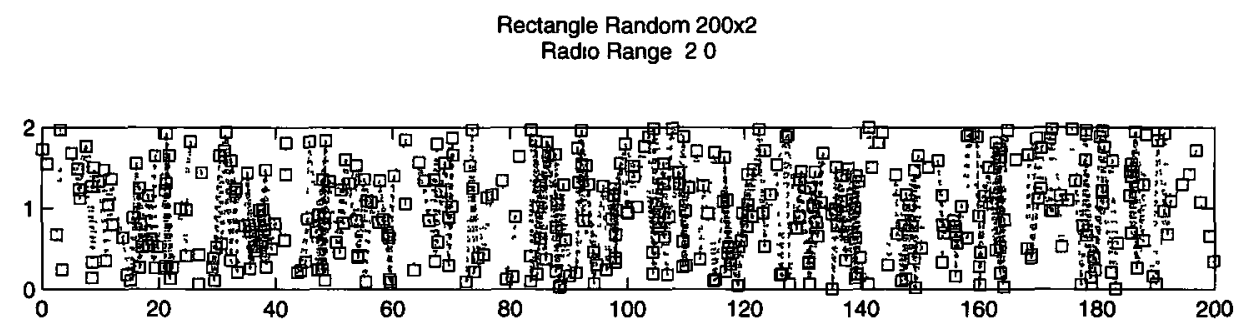

Figure 6.4: Example of pipeline network topology

the floor of possible localization errors drops. In particular, when the network depth is only 1 , the floor is about $0.5 \mathrm{r}$, whereas when the depth is 4 units, or twice the radio range, the floor drops to roughly $0.25 \mathrm{r}$. Also, as the sum of the distance increases and the network depth is greater than the radio range, the outlier cases become apparent.

Interestingly, the outlier cases have a much lower mean localization error in a pipeline topology. Figure 6.6 shows two sample anchor sets that demonstrate the difference in localization performance when the anchors are clumped together versus spread apart. When the anchor nodes are clumped together, the calculated positions remain in a straight line, as the expected results do, but the algorithm cannot determine the correct angle that line should take. However, since the lines do cross where the anchor nodes are clumped, the line is grounded to a relatively accurate level, when compared with the square networks. For this reason, the outlier case, in general, has lower error in a pipeline topology. Nonetheless, it is poor performance, and is likely not useful in most applications.

The plots of minimum triangle height in Figure 6.7 show that for networks with a depth less than the radio range, the separation of good and outlier cases is impossible to distinguish. Specifically, when the network depth is 1 units, half the radio range, the mean localization error is basically flat across all triangle heights possible. As it becomes possible to have anchor sets forming a triangle with height more than the radio range, localization performance drops down to normal levels, below $0.5 r$, 

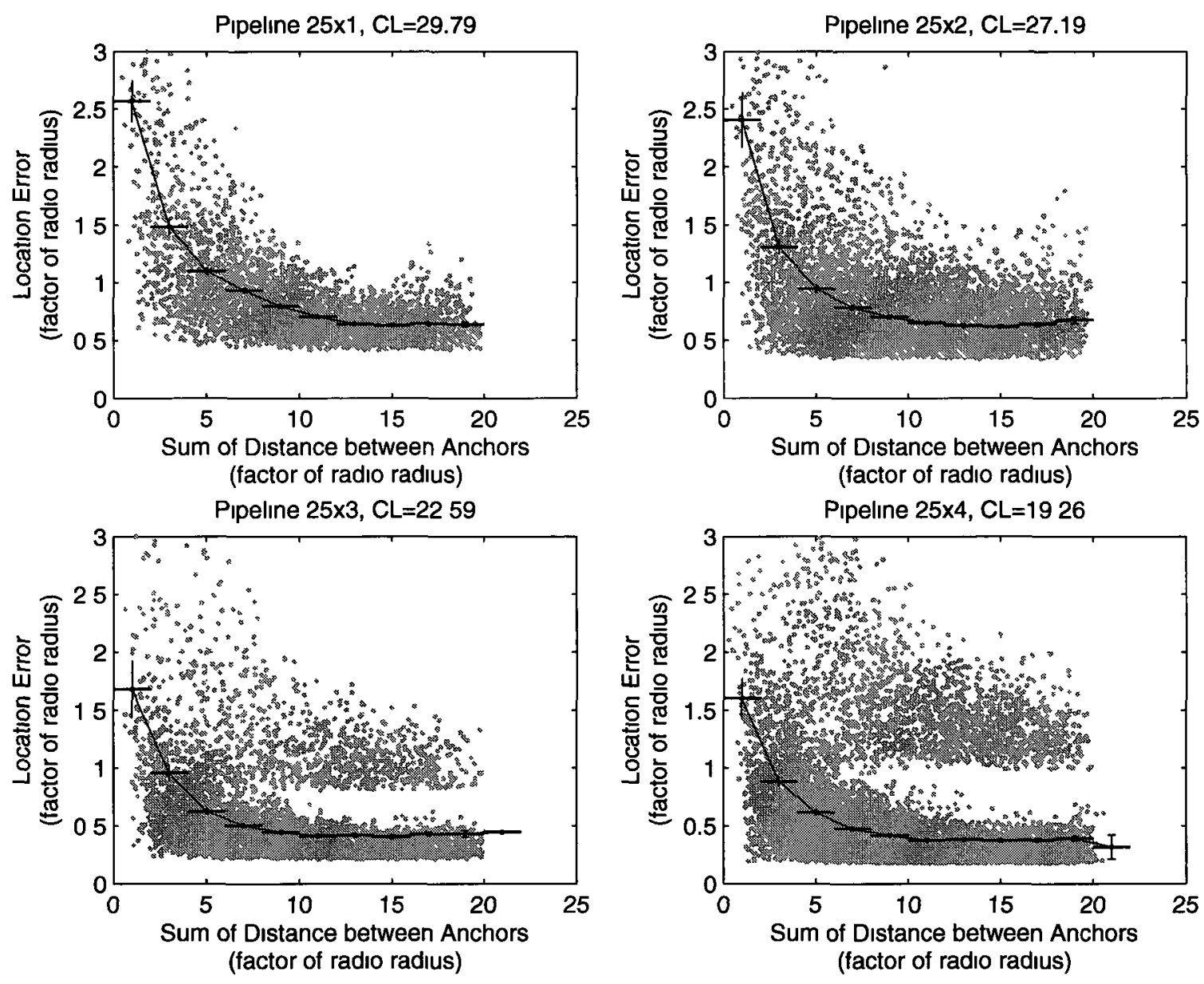

Figure 6.5: Sum of distance between anchors vs. mean location error for random pipeline 25-by-1,2,3 and 4 unit networks with 200 nodes, 1,000 anchor sets each, and a radio range of 2.0 units 


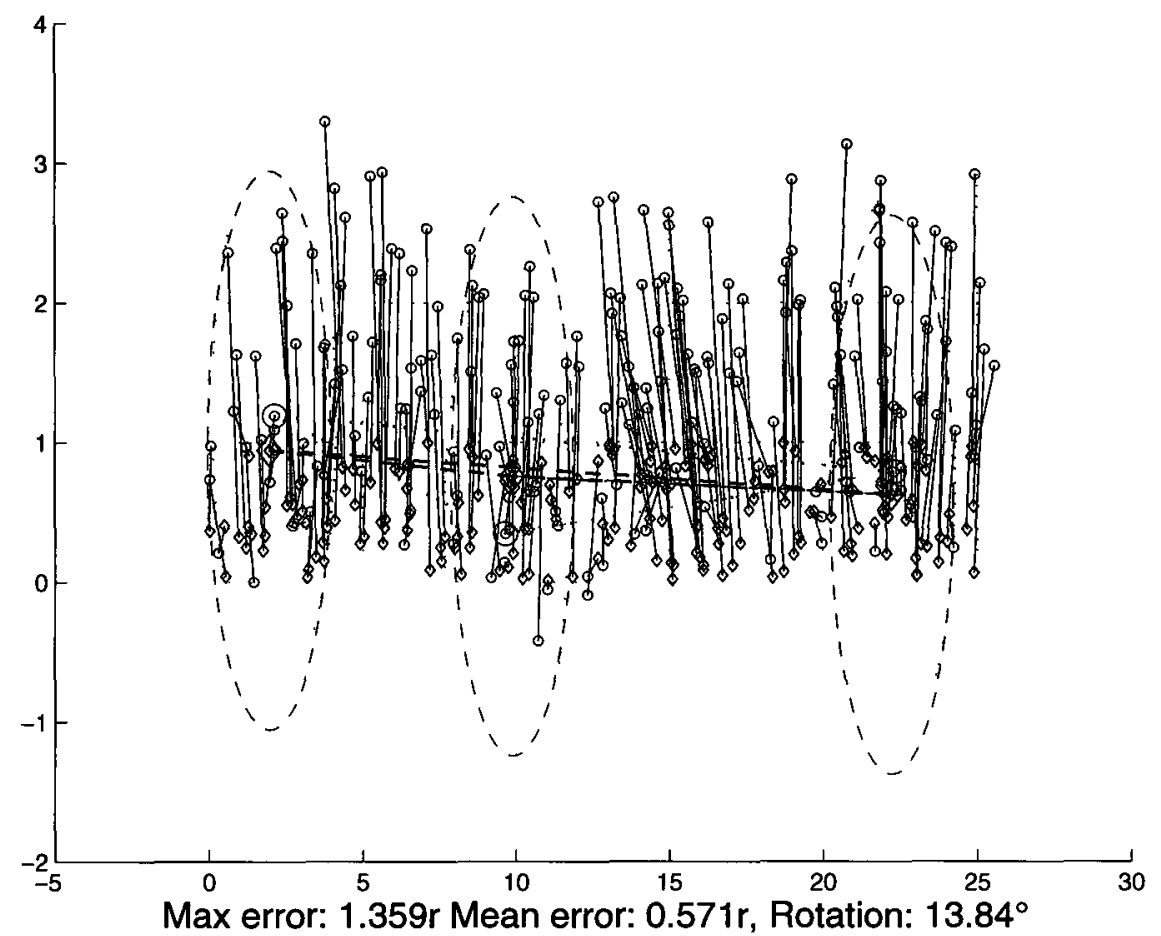

(a) Pipeline with spread apart anchors

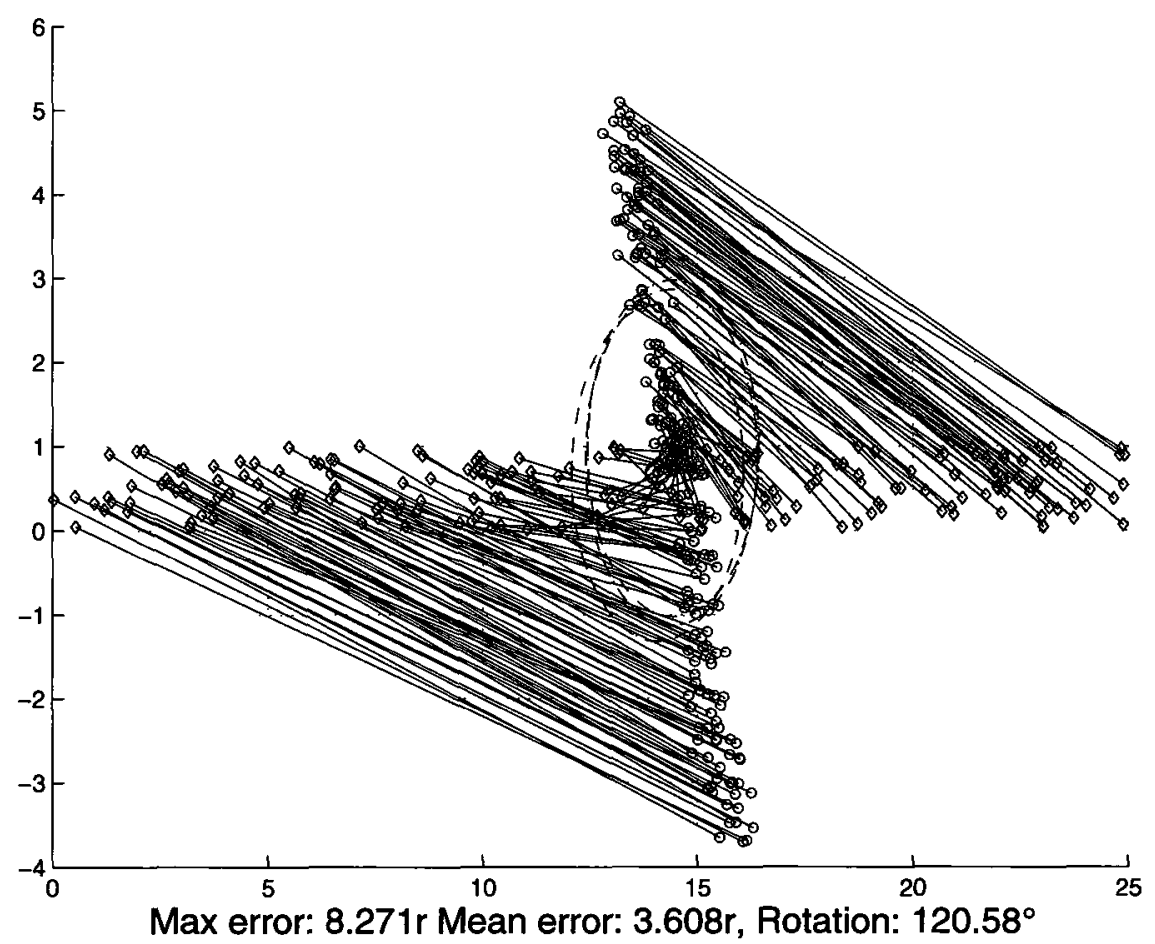

(b) Pipeline with clumped anchors

Figure 6.6: Pipeline network with anchors spread apart and clumped together 

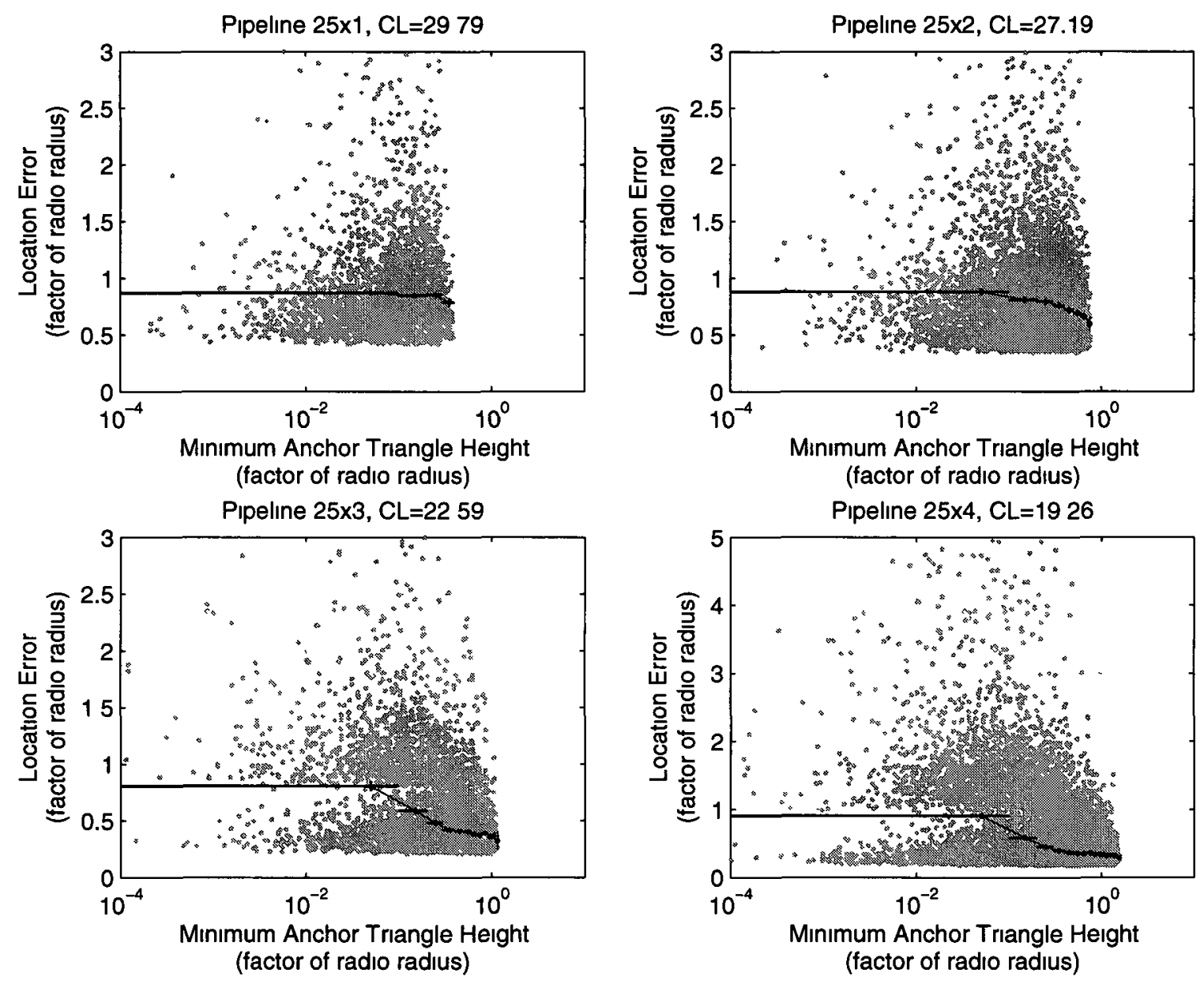

Figure 6.7: Minimum height of anchor triangle vs. mean location error for random pipeline 25-by-1,2,3 and 4 unit networks with 200 nodes, 1,000 anchor sets each, and a radio range of 2.0 units

when the triangle height is also greater than the radio range. However, the minimum triangle height no longer appears to be a good indicator of outlier cases.

In the end, even though the results are better when the depth of the pipeline is more than the radio range, it is impossible to reliably avoid the outlier condition. Therefore, for a pipeline topology, we recommend to use a different class of localization algorithms that does not rely on transforming local coordinates into global coordinates with anchor nodes. If no other algorithms are possible, we recommend making the 
network as deep as possible and to spread the anchors as apart along the length of the pipeline as possible. 


\section{Chapter 7}

\section{Other Factors}

So far, we have examined anchor placement as the sole factor impacting localization error. We set out here to show that there are indeed other factors that play a significant role in determining localization performance.

In the following example, the same anchor set is used in four different random networks. Figure 7.1 shows two non-outlier cases. The mean error for each network, despite using the same anchor set, is different. What is surprising is the degree to which they are different: $37 \%$. Figure 7.2 shows two more networks with the same anchor set. This time, both of the plots reveal outlier cases. Despite using the same anchor set, the localization performance differs significantly. While we have shown that anchor placement does play a role in the localization performance, these simple

examples clearly show that there are significant other factors affecting the localization error.

These results show that there is significant variation in localization results across networks, when the anchor set is geographically fixed. In other words, the absolute anchor positions are not enough to predict localization errors. Therefore, it is clear there are other factors besides anchor placement effecting the resulting location errors. A key piece of future work is to isolate and analyze these other factors. However, 


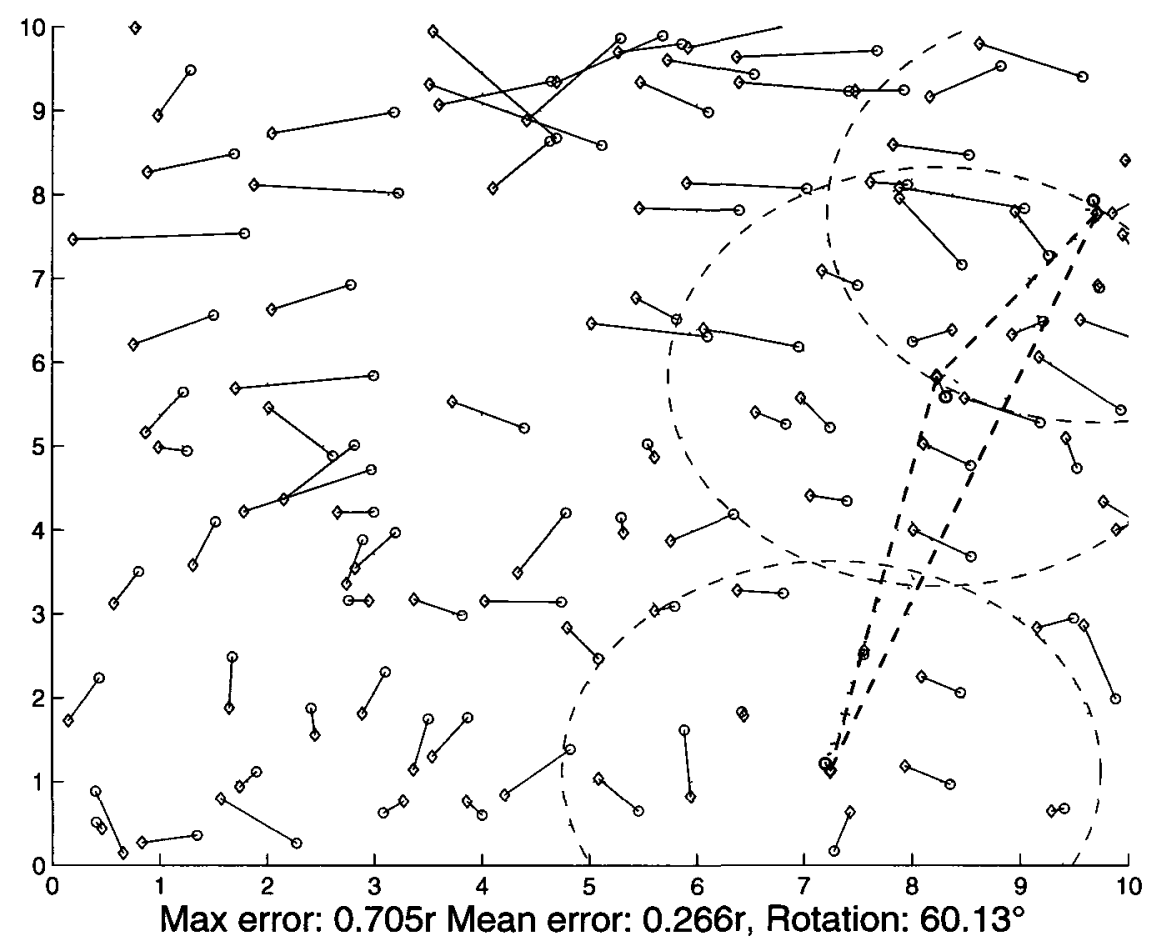

(a) Network A

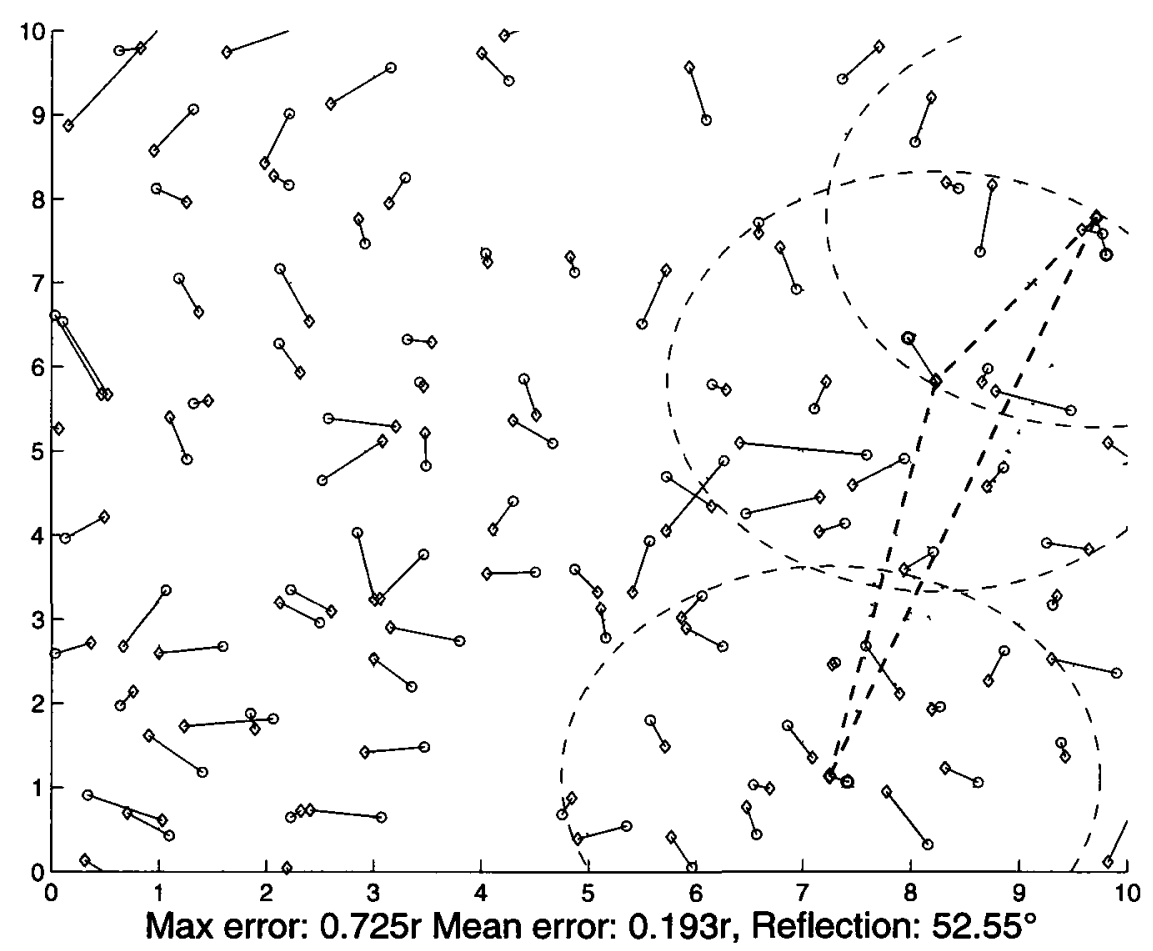

(b) Network B

Figure 7.1: Good localization performance with same anchor set in two different networks 


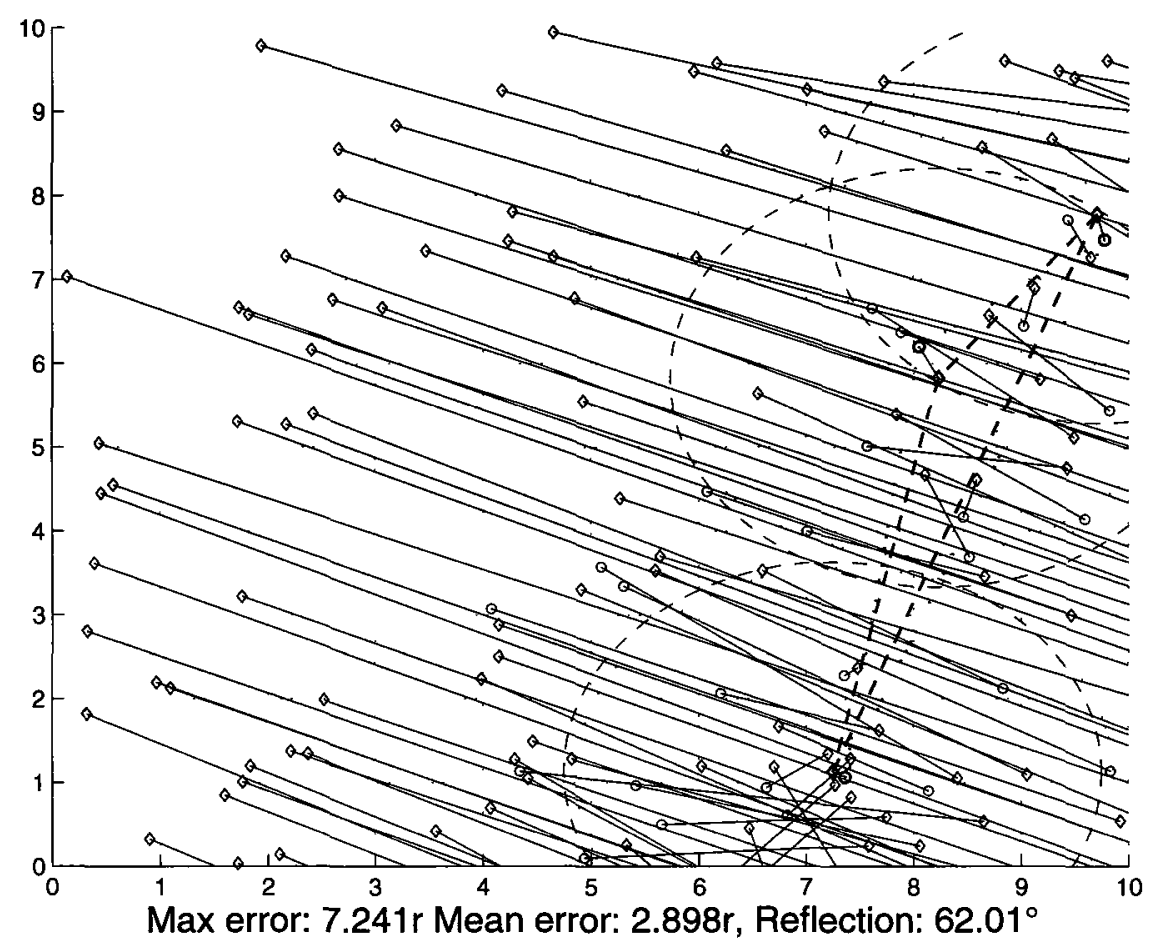

(a) Network C

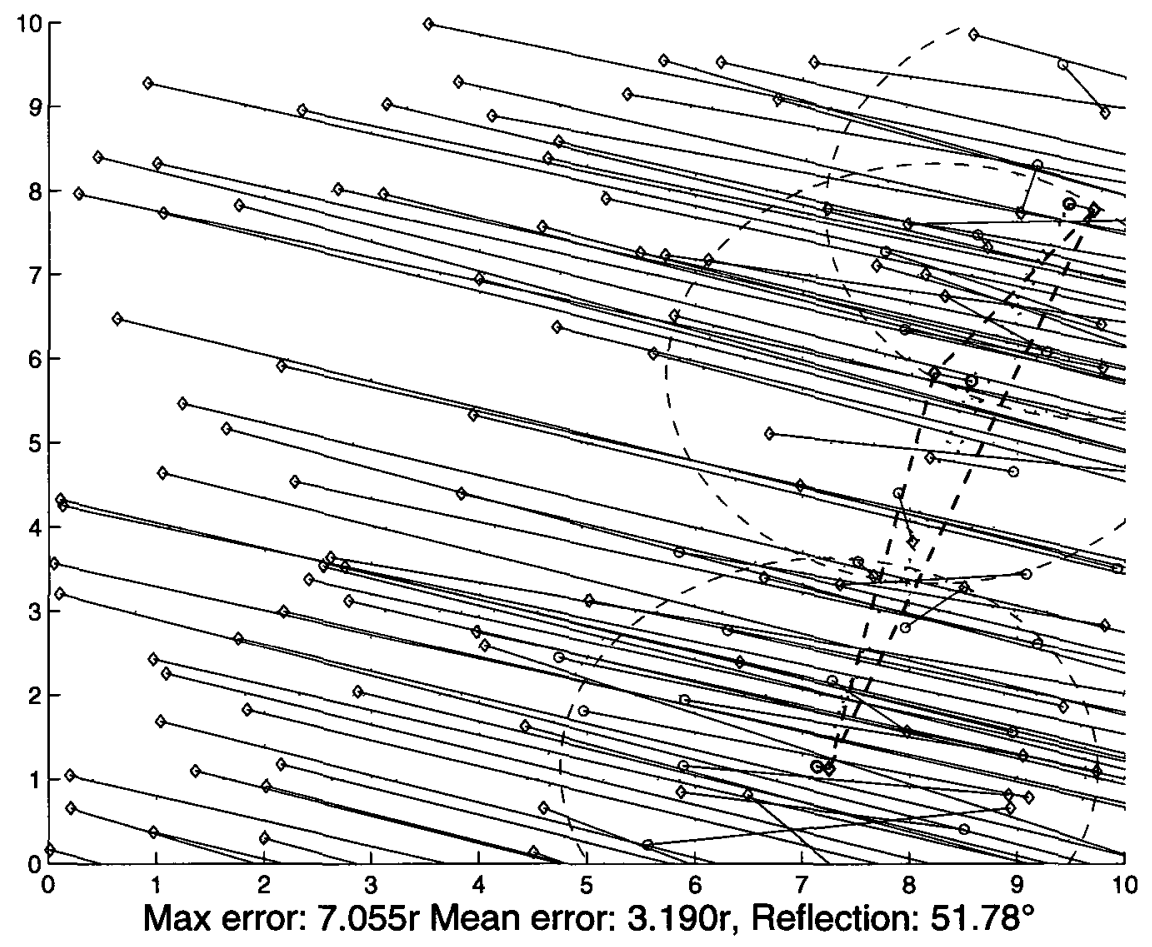

(b) Network D

Figure 7.2: Poor localization performance with same anchor set in two different networks 
since the outlier case itself is undetectable in the absence of extensive ground truth data, the best we can hope for is a way to minimize the probability beyond analyzing the height of the anchor node triangle by studying what these other factors might be.

Some of these factors have been previously explored. Network connectivity level is highlighted in [?]. Connectivity level is a factor of both node density and radio range. During this work, we discovered the node chosen to start the local map patching also has an effect on the end result, although not always significant. Another option worth exploring is whether different deployment options of CCA-MAP will impact the mean localization accuracy. For example, as described in [?], very accurate localization results are possible if neighborhood information of all nodes is collected centrally and CCA-MAP applied to the global connectivity matrix. Given the inherent flexibility in how CCA-MAP can be deployed, calculating relative local maps at each node, at some cluster heads, or centrally, may result in significantly different mean localization errors. 


\section{Chapter 8}

\section{Conclusion}

\subsection{Summary and Contribution}

This study provides two key guidelines for network designers and users of wireless sensor networks when choosing anchor node positions or assessing the quality of localization results. Namely, make sure that the sum of the distance between anchor nodes is at least ten times the radio range and that the minimum height of the triangle formed by the anchor nodes is at least equal to the radio range. Further, the larger these two metrics are, the lower the mean location error of the network will be, on average, and the lower the probability of using an anchor set that will cause extremely poor localization performance. Effectively, this means do not put the anchor nodes in a straight line or close to each other. We have further shown that these criteria apply to network topologies where the overall network area is two-dimensional, but fails when the network topology approaches one-dimension, meaning it is extremely narrow compared with the radio range, for example when monitoring pipelines or roads.

While the simulations in this study use the CCA-MAP algorithm, the results apply to any protocol in which a local coordinate system is transformed into a global 
coordinate system using a set of anchor nodes with Procrustes analysis. Further, the scope of the study extends beyond wireless sensor network protocols and can be applied to any transformation problem where a small subset of points is used as the basis to transform a set of points.

\subsection{Future Work}

While we have provided some recommendations for anchor placement, there are a few key areas where future study could enhance the results. First, an examination of the other factors affecting localization performance would be beneficial, as described in Chapter 7. Specifically, analysis of the results presented here, as they are affected by network connectivity levels. A raw examination of other as of yet undiscovered factors could also uncover ways to further improve localization accuracy. Second, expanding these results to three-dimensions may benefit some network designers. For example, a sensor network may be deployed through a high-rise building or on a bridge. While we expect that results to be similar, it is worthwhile to confirm. Outside the scope of sensor networks and the physical world, these results could also apply to any dimension of data. This would effectively be an exhaustive study of Procrustes analysis where a set of data in one coordinate system is transformed into another coordinate system based on a subset of known points. Last, finding other methodologies besides Procrustes analysis to provide a transformation between local and global coordinates. This may be especially beneficial if applied to pipeline topologies specifically. 


\section{List of References}

[1] D. Nicelescu and B. Nath, "Ad Hoc Positioning System (APS)," in Global Telecommunications Conference (GLOBECOM 2001), vol. 5, (San Antonio, Texas), pp. 2926-2931, November 2001.

[2] Y. Shang, W. Rumi, Y. Zhang, and M. Fromherz, "Localization from connectivity in sensor networks," Parallel and Distributed Systems, IEEE Transactıons on, vol. 15, pp. 961-974, November 2004.

[3] L. Li and T. Kunz, "Localization applying an efficient neural network mapping," in Proceedings of the 1st international conference on Autonomic computing and communication systems (Autonomics 2007), vol. 302, (Rome, Italy), 2007.

[4] "Procrustes analysis." http://www.mathworks.com/help/toolbox/stats/ procrustes.html, 2010.

[5] L. Li and T. Kunz, "Cooperative node localization using nonlinear data projection," ACM Transactions on Sensor Networks (TOSN), vol. 5, February 2009.

[6] J. Kumagai, "The secret life of birds," IEEE Spectrum, vol. 41, pp. 42-48, April 2004.

[7] "RUNES (Reconfigurable Ubiquitous Networked Embedded Systems) IST Project." http://www. ist-runes.org, 2004-2006.

[8] B. Wellenhoff, H. Lictenegger, and J. Collins, Global Posıtıonıng System: Theory and Practıce. Berlin, Germany: Springer, 4th ed., 1997.

[9] A. Savvides, C. Han, and M. Strivastava, "Dynamic finegrained localization in ad-hoc networks of sensors," in Proceedings of the 7th Annual International Conference on Mobıle Computing and Networking (MobıCom'01, (Rome, Italy), pp. 166-169, July 2001. 
[10] D. Nicelescu and B. Nath, "Ad hoc positioning system (APS) using AOA," in Twenty-Second Annual Joint Conference of the IEEE Computer and Communications (INFOCOM 2003), vol. 3, (San Francisco, California), pp. 1734-1743, April 2003.

[11] N. Patwari, A. Hero, M. Perkins, N. Correal, and R. O'Dea, "Relative location estimation in wireless sensor networks," IEEE Transactıons on Signal Processing, vol. 51, no. 8, pp. 2137-2148, 2003.

[12] Y. Shang and W. Ruml, "Improved mds-based localization," in INFOCOM 2004. Twenty-thırd AnnualJoint Conference of the IEEE Computer and Communications Socretıes, vol. 4, pp. 2640-2651, March 2004.

[13] P. Demartines and J. Herault, "Curvilinear component analysis: a self-organizing neural network for nonlinear mapping of data sets," Neural Networks, IEEE Transactions on, vol. 8, pp. 148-154, January 1997.

[14] L. Doherty, K. Pister, and L. El Ghaoui, "Convex position estimation in wireless sensor networks," in INFOCOM 2001. Twentreth Annual Joint Conference of

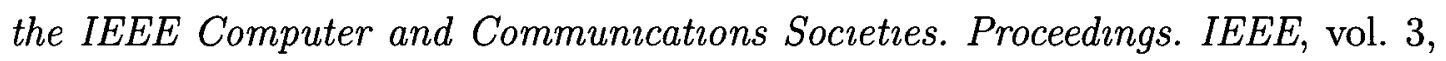
pp. 1655-1663, 2001.

[15] S. Hara and T. Fukumura, "Determination of the placement of anchor nodes satisfying a required localization accuracy," in Wireless Communicatıon Systems. 2008. ISWCS '08. IEEE Internatıonal Symposıum on, pp. 128-132, October 2008.

[16] J. Ash and R. Moses, "On optimal anchor node placement in sensor localization by optimization of subspace principal angles," in Acoustics, Speech and Signal Processing, 2008. ICASSP 2008. IEEE Internatıonal Conference on, pp. 22892292, March 2008.

[17] H. Karl and A. Willig, Protocols and Architectures for Wireless Sensor Networks. John Wiley \& Sons, Ltd, 2005.

[18] C. Savarese, J. M. Rabaey, and K. Langendoen, "Robust positioning algorithms for distributed ad-hoc wireless sensor networks," in ATEC '02: Proceedings of the General Track of the annual conference on USENIX Annual Technical Conference, (Berkeley, CA, USA), pp. 317-327, USENIX Association, 2002. 
[19] K.-Y. Cheng, K.-S. Lui, and V. Tam, "Hybloc: Localization in sensor networks with adverse anchor placement," Sensors, vol. 9, no. 1, pp. 253-280, 2009.

[20] H. Lim and J. Hou, "Localization for anisotropic sensor networks," vol. 1, pp. 138-149, March 2005.

[21] R. Zemek, M. Takashima, S. Hara, K. Yanagihara, K. Fukui, S. Fukunaga, and K. Kitayama, "An effect of anchor nodes placement on a target location estimation performance," in TENCON 2006. 2006 IEEE Regıon 10 Conference, pp. 1-4, November 2006. 


\section{Appendix A}

\section{Matlab@Simulation Code}

All source code used to simulate CCA-MAP and generate resulting data can be found in Google(c)Code. The code was originally written by $\mathrm{Li} \mathrm{Li}$ and modified for the purposes of readability and different output data and plots.

The primary files are:

- ccaconfig.m: Property file to setup the network shape, number of anchor sets, anchor placement, radio range and other simulation parameters.

- simcca.m: Generate and simulate a network over the configured number of anchor sets and output a suite of statistical plots.

- multisimcca.m: Generate and simulate a configured number of networks, with the same exact anchor sets and output a suite of statistical plots.

- indicator.m: Outputs a suite of statistical plots for a set of previously run output directories from simcca.m. This allows combining multiple networks and their anchor sets into a single analysis.

See http://code.google.com/p/sim4j/source/browse/\#svn/trunk/thesis/ matlab for details. 